\title{
LOGARITHMS AND SECTORIAL PROJECTIONS FOR ELLIPTIC BOUNDARY PROBLEMS
}

\author{
ANDERS GAARDE and GERD GRUBB
}

\begin{abstract}
On a compact manifold with boundary, consider the realization $B$ of an elliptic, possibly pseudodifferential, boundary value problem having a spectral cut (a ray free of eigenvalues), say $R_{-}$. In the first part of the paper we define and discuss in detail the operator $\log B$; its residue (generalizing the Wodzicki residue) is essentially proportional to the zeta function value at zero, $\zeta(B, 0)$, and it enters in an important way in studies of composed zeta functions $\zeta(A, B, s)=\operatorname{Tr}\left(A B^{-s}\right)$ (pursued elsewhere).

There is a similar definition of the operator $\log _{\theta} B$, when the spectral cut is at a general angle $\theta$. When $B$ has spectral cuts at two angles $\theta<\varphi$, one can define the sectorial projection $\Pi_{\theta, \varphi}(B)$ whose range contains the generalized eigenspaces for eigenvalues with argument in $] \theta, \varphi[$; this is studied in the last part of the paper. The operator $\Pi_{\theta, \varphi}(B)$ is shown to be proportional to the difference between $\log _{\theta} B$ and $\log _{\varphi} B$, having slightly better symbol properties than they have. We show by examples that it belongs to the Boutet de Monvel calculus in many special cases, but lies outside the calculus in general.
\end{abstract}

\section{Introduction}

The purpose of this paper is to set up logarithms and sectorial projections for elliptic boundary value problems, and to establish and analyze residue definitions associated with these operators. Let us first recall the situation for boundaryless manifolds:

For a classical elliptic pseudodifferential operator $(\psi$ do) $P$ of order $m>0$, acting in a vector bundle $\widetilde{E}$ over a closed (i.e., compact boundaryless) $n$ dimensional manifold $\widetilde{X}$, certain functions of the operator have been studied with great interest for many years. Assuming that $P$ has no eigenvalues on some ray, say $\mathbf{R}_{-}$, one has from Seeley's work [16] that the complex powers $P^{-s}$ can be defined as $\psi$ do's by use of the resolvent $(P-\lambda)^{-1}$. Moreover, the zeta function $\zeta(P, s)=\operatorname{Tr}\left(P^{-s}\right)$ has a meromorphic extension to $s \in \mathrm{C}$ with at most simple poles at the real numbers $\{(n-j) / m \mid j \in \mathrm{N}\}$ (we denote $\{0,1,2, \ldots\}=\mathrm{N})$. There is no pole at $s=0($ for $j=n)$, and the value $\zeta(P, 0)$ plays an important role in index formulas. Let us define the basic zeta value

\footnotetext{
Received March 27, 2007.
} 
$C_{0}(P)$ by

$$
C_{0}(P)=\zeta(P, 0)+v_{0},
$$

where $\nu_{0}$ is the algebraic multiplicity of the zero eigenvalue of $P$ (if any). It is well-known how $C_{0}(P)$ can be calculated in local coordinates from finitely many homogeneous terms of the symbol of $P$.

Another interesting function of $P$ is $\log P$, defined on smooth functions by

$$
\log P=\lim _{s \downarrow 0} \frac{i}{2 \pi} \int_{\mathscr{C}} \lambda^{-s} \log \lambda(P-\lambda)^{-1} d \lambda ;
$$

here $\lambda^{-s}$ and $\log \lambda$ are taken with branch cut $\mathbf{R}_{-}$, and $\mathscr{C}$ is a contour in $C \backslash \overline{\mathbf{R}}_{-}$ going around the nonzero spectrum of $P$ in the positive direction. By use of the fact that $\log P=-\left.\frac{d}{d s} P^{-s}\right|_{s=0}$, Scott [15] showed that

$$
C_{0}(P)=-\frac{1}{m} \operatorname{res}(\log P),
$$

where $\operatorname{res}(\log P)$ is a slight generalization of Wodzicki's noncommutative residue ([20], Guillemin [11]).

In the case of a compact $n$-dimensional manifold $X$ with boundary $\partial X=X^{\prime}$ (smoothly imbedded in an $n$-dimensional manifold $\widetilde{X}$ without boundary), one can study the analogous operators and constants defined from a realization $B$ of a pseudodifferential (or differential) elliptic boundary value problem. Here $B=(P+G)_{T}$, defined from a system $\left\{P_{+}+G, T\right\}$ of order $m>0(m \in \mathrm{Z})$ in the Boutet de Monvel calculus [2], where $P$ is a $\psi$ do on $\widetilde{X}$ and $P_{+}$is its truncation to $X$ (acting in $E=\left.\widetilde{E}\right|_{X}$ ), $G$ is a singular Green operator (s.g.o.) and $T$ is a system of trace operators. $B$ is the operator acting like $P_{+}+G$ with domain

$$
D(B)=\left\{u \in H^{m}(X, E) \mid T u=0\right\},
$$

where $H^{m}(X, E)$ is the Sobolev space of order $m$. In the differential operator case, $G=0$. Assuming that for $\lambda$ on a ray, say $\mathrm{R}_{-},\left\{P_{+}+G-\lambda, T\right\}$ satisfies the hypotheses of parameter-ellipticity of Grubb [6, Sect. 3.3] (consistent with those of Seeley [17] in the differential operator case), one can define the complex powers by functional analysis and study the pole structure of $\zeta(B, s)=\operatorname{Tr}\left(B^{-s}\right)$ [6, Sect. 4.4], and in particular discuss the basic zeta value $C_{0}(B)$ defined similarly to (1.1). However, in contrast with the closed manifold case, the powers $B^{-s}$ do not lie in the calculus we are using (in particular their $\psi$ do part does not satisfy the transmission condition of [2]). Then it is advantageous to build the analysis more directly on the resolvent, which does belong to the parameter-dependent calculus set up in [6]. In fact, for $N>n / m$ 
(such that $(B-\lambda)^{-N}$ is trace-class), there is a trace expansion for $\lambda \rightarrow \infty$ in a sector $V$ around $\mathbf{R}_{-}$:

$$
\operatorname{Tr}(B-\lambda)^{-N}=\sum_{0 \leq j \leq n} c_{j}^{(N)}(-\lambda)^{(n-j) / m-N}+O\left(\lambda^{-N-\varepsilon}\right)
$$

$(\varepsilon>0)$, and here

$$
C_{0}(B)=c_{n}^{(N)},
$$

independently of $N$. It is shown in [8] that for a generalization of (1.3) to $B$,

$$
C_{0}(B)=-\frac{1}{m} \operatorname{res}(\log B),
$$

it is sufficient to be able to define $\log B$; the complex powers $B^{-s}$ are not needed.

The present paper gives in Sections 2 and 3 a detailed study of $\log B$. For one thing, this allows a more precise interpretation of the formula (1.7), initiated in [8]. Another important purpose is to open up for the use of compositions of $\log B$ with other operators. These are needed for the consideration of composed zeta functions $\zeta(A, B, s)=\operatorname{Tr}\left(A B^{-s}\right)$ with general $A$ from the calculus of [2], or rather, trace expansion formulas for composed resolvents $A(B-\lambda)^{-N}$. Such a study is carried out in [9] using the results on $\log B$ obtained in the present paper. We show in Section 2 that

$$
\log B=(\log P)_{+}+G^{\log },
$$

where $G^{\log }$ is a generalized singular Green operator satisfying a specific part of the usual symbol estimates for s.g.o.s; its principal part has a singularity at the boundary. In Section 3 we study its residue.

If, more generally than $\mathrm{R}_{-}$, the ray free of eigenvalues for $B$ (the spectral cut) is $e^{i \theta} \mathbf{R}_{+}$for some angle $\theta$, the corresponding operator functions will be defined by formulas where $\lambda^{-s}$ and $\log \lambda$ (as in (1.2)) are replaced by $\lambda_{\theta}^{-s}$ and $\log _{\theta} \lambda$ with branch cut $e^{i \theta} \mathbf{R}_{+}$, and the integration curve runs in $C \backslash e^{i \theta} \overline{\mathbf{R}}_{+}$. The functions are then provided with an index $\theta$;

$$
\zeta_{\theta}(B, s)=\operatorname{Tr}\left(B_{\theta}^{-s}\right), \quad \log _{\theta} B=\left(\log _{\theta} P\right)_{+}+G^{\log _{\theta}} .
$$

When $B$ has spectral cuts at $\theta$ and $\varphi$ for some $\theta<\varphi<\theta+2 \pi$, it is of interest to study the sectorial projection $\Pi_{\theta, \varphi}(B)$, a projection whose range contains the generalized eigenspace of $B$ for the sector $\Lambda_{\theta, \varphi}=\left\{r e^{i \omega} \mid r>0, \theta<\omega<\varphi\right\}$ and whose nullspace contains the generalized eigenspace of $B$ for $\Lambda_{\varphi, \theta+2 \pi}$; it was considered earlier by Burak [3], and in the boundaryless case by Wodzicki 
[20], Ponge [14]. We show in Section 4 that it equals $\frac{i}{2 \pi}\left(\log _{\theta} B-\log _{\varphi} B\right)$ and has the form

$$
\Pi_{\theta, \varphi}(B)=\left(\Pi_{\theta, \varphi}(P)\right)_{+}+G_{\theta, \varphi} .
$$

Here $\Pi_{\theta, \varphi}(P)$ is a zero-order classical $\psi$ do, which satisfies the transmission condition when $m$ is even, and $G_{\theta, \varphi}$ is a generalized s.g.o., bounded in $L_{2}$ in the differential operator case. There are natural types of examples where $G_{\theta, \varphi}$ is a standard s.g.o. as in [2], but in general it will be of a generalized type satisfying only part of the standard symbol estimates.

We expect to take up elsewhere the study of its residue, whose possible vanishing is important for the study of eta functions associated with $B$.

\section{The singular Green part of the logarithm}

Let $X$ be a compact $n$-dimensional $C^{\infty}$ manifold with boundary $\partial X=X^{\prime}$, provided with a hermitian $C^{\infty}$ vector bundle $E$. We can assume that $X$ is smoothly imbedded in an $n$-dimensional manifold $\widetilde{X}$ without boundary and that $E$ is the restriction to $X$ of a bundle $\widetilde{E}$ over $\widetilde{X}$. Consider a system $\left\{P_{+}+G, T\right\}$ of operators in the Boutet de Monvel calculus [2] (pseudodifferential boundary operators, $\psi$ dbo's). Here $P$ is defined as a $\psi$ do of order $m>0$ on $\widetilde{X}$ acting on the sections of $\widetilde{E}$, and its truncation to $X$ is

$$
P_{+}=r^{+} P e^{+}, \quad r^{+} \text {restricts from } \tilde{X} \text { to } X^{\circ}, e^{+} \text {extends by } 0 .
$$

To assure that $P_{+}$maps $C^{\infty}(X, E)$ into itself, $P$ is assumed to satisfy the transmission condition, which means that in local coordinate systems at the boundary, where the manifold is replaced by $\mathbf{R}_{+}^{n}=\left\{x=\left(x_{1}, \ldots, x_{n}\right) \mid x_{n}>\right.$ $0\}$, with notation $x^{\prime}=\left(x_{1}, \ldots, x_{n-1}\right)$,

$$
\begin{aligned}
& \partial_{x}^{\beta} \partial_{\xi}^{\alpha} p_{m-j}\left(x^{\prime}, 0,0,-\xi_{n}\right) \\
& \quad=(-1)^{m-j-|\alpha|} \partial_{x}^{\beta} \partial_{\xi}^{\alpha} p_{m-j}\left(x^{\prime}, 0,0, \xi_{n}\right) \quad \text { for } \quad\left|\xi_{n}\right| \geq 1,
\end{aligned}
$$

for all indices; $m$ is integer. (A discussion of such conditions can be found in Grubb and Hörmander [10].) $G$ is a singular Green operator in $E$ of order and class $m$, and $T=\left\{T_{0}, \ldots, T_{m-1}\right\}$ is a system of trace operators $T_{k}$ of order and class $k$, going from $E$ to bundles $F_{k}$ over $\partial X$, defining an elliptic boundary value problem. In particular,

$$
\sum_{0 \leq k \leq m-1} \operatorname{dim} F_{k}=\frac{1}{2} m \operatorname{dim} E .
$$

Details on these operator types can be found in [2], [6]. 
We assume that the system $\left\{P_{+}+G-\lambda, T\right\}$ satisfies the conditions of parameter-ellipticity in [6, Def. 3.3.1] for $\lambda$ on the rays in a sector $V$ around $\mathrm{R}_{-}$. In particular, it can be a differential operator system; here $P$ and $T$ are differential, and $G$ is omitted. A classical example is the Laplace operator on a domain in $\mathrm{R}^{n}$, together with the Dirichlet trace operator $T=\gamma_{0}$.

It should be noted that the hypotheses imply that the trace operator is normal, as accounted for in [6, Section 1.5].

The system has a certain regularity number $v$ in the sense of [6]; it is an integer or half-integer in $\left[\frac{1}{2}, m\right]$ for pseudodifferential problems, $+\infty$ for purely differential problems.

From the system we define the realization $B=(P+G)_{T}$ as the operator acting like $P_{+}+G$ with domain (1.4). By [6, Ch. 3], the resolvent $R_{\lambda}=$ $(B-\lambda)^{-1}$ exists on each ray in $V$ for sufficiently large $|\lambda|$, and is $O\left(\lambda^{-1}\right)$ in $L_{2}$ operator norm there. It has the structure

$$
R_{\lambda}=Q_{\lambda,+}+G_{\lambda},
$$

where $Q_{\lambda}=(P-\lambda)^{-1}$ on $\widetilde{X}$ (which can be assumed to be compact), and $G_{\lambda}$ is the singular Green part. Since the spectrum of $B$ is discrete, we can assume (after a small rotation if necessary) that $\mathbf{R}_{-}$is free of eigenvalues of $B$, and likewise for $P$.

We shall define the operator $\log (B)=\log \left((P+G)_{T}\right)$, also written $\log B$, $\log (P+G)_{T}$, by

$$
\log (P+G)_{T}=\lim _{s \searrow 0} \frac{i}{2 \pi} \int_{\mathscr{C}} \lambda^{-s} \log \lambda R_{\lambda} d \lambda,
$$

to be further explained below; here $\mathscr{C}$ is a Laurent loop

$$
\mathscr{C}=\left\{r e^{i \pi} \mid \infty>r>r_{0}\right\} \cup\left\{r_{0} e^{i \omega} \mid \pi \geq \omega \geq-\pi\right\} \cup\left\{r e^{-i \pi} \mid r_{0}<r<\infty\right\}
$$

going around the nonzero spectrum of $(P+G)_{T}$ in the positive direction.

Insertion of the decomposition (2.4) in the defining formula (2.5) shows that $Q_{\lambda,+}$ contributes with

$$
\lim _{s \searrow 0} \frac{i}{2 \pi} \int_{\mathscr{C}} \lambda^{-s} \log \lambda r^{+} Q_{\lambda} e^{+} d \lambda=r^{+}(\log P) e^{+}=(\log P)_{+},
$$

where $\log P$ is well-known from the closed manifold case, cf. (1.2). Its symbol in local coordinates is of the form

$$
\operatorname{symb}(\log P)=m \log [\xi]+l(x, \xi),
$$


where $l(x, \xi)$ is a classical $\psi$ do symbol of order 0 (see also the lemma below), and $[\xi]$ is a smooth positive function that equals $|\xi|$ for $|\xi| \geq 1$. The operator is continuous from $H^{t}(\widetilde{X}, \widetilde{E})$ to $H^{t-\varepsilon}(\widetilde{X}, \widetilde{E})$ for any $\varepsilon>0$; hence

$$
(\log P)_{+}: H^{t}(X, E) \rightarrow H^{t-\varepsilon}(X, E) \quad \text { for } \quad|t|<\frac{1}{2} .
$$

(The limit for $s \rightarrow 0$ in (2.7) can be taken in this operator norm.)

In even-order cases, the transmission condition satisfied by $P$ carries over to $l(x, \xi)$ :

Lemma 2.1. When $m$ is even, $l(x, \xi)$ satisfies the transmission condition.

Proof. As shown e.g. in Okikiolu [13], the symbol of $\log P$ is calculated in local coordinates from the symbol $q(x, \xi, \lambda)$ of $Q_{\lambda}$ by integration with $\log \lambda$ around the spectrum of the principal symbol $p_{m}$ of $P$; here the quasihomogeneous terms in the expansion $q(x, \xi, \lambda) \sim \sum_{j \in \mathrm{N}} q_{-m-j}(x, \xi, \lambda)$ (homogeneous of degree $-m-j$ in $\left(\xi,|\lambda|^{\frac{1}{m}}\right)$ on each ray) contribute as follows:

$$
\begin{aligned}
& \frac{i}{2 \pi} \int_{\mathscr{C}(x, \xi)} \log \lambda q_{-m}(x, \xi, \lambda) d \lambda \\
& \quad=\frac{i}{2 \pi} \int_{\mathscr{C}(x, \xi)} \log \lambda\left(p_{m}(x, \xi)-\lambda\right)^{-1} d \lambda=\log p_{m}(x, \xi) \\
& \quad=\log \left([\xi]^{m}\right)+\log \left([\xi]^{-m} p_{m}(x, \xi)\right)=m \log [\xi]+l_{0}(x, \xi), \\
& \frac{i}{2 \pi} \int_{\mathscr{C}(x, \xi)} \log \lambda q_{-m-j}(x, \xi, \lambda) d \lambda=l_{-j}(x, \xi) \quad \text { for } \quad j>0,
\end{aligned}
$$

where $\mathscr{C}(x, \xi)$ is a closed curve in $C \backslash \bar{R}_{-}$around the spectrum of $p_{m}(x, \xi)$. Each $l_{-j}$ is homogeneous in $\xi$ of degree $-j$ for $|\xi| \geq 1$; for $j=0$ it follows since $[\xi]^{-m} p_{m}(x, \xi)$ is so, and for $j \geq 1$ it is seen e.g. as follows (where we set $\lambda=t^{m} \varrho$ ):

$$
\begin{aligned}
l_{-j}(x, t \xi) & =\frac{i}{2 \pi} \int_{\mathscr{C}(x, t \xi)} \log \lambda q_{-m-j}(x, t \xi, \lambda) d \lambda \\
& =\frac{i}{2 \pi} \int_{t^{-m} \mathscr{C}(x, t \xi)}(\log \varrho+m \log t) t^{-m-j} q_{-m-j}(x, \xi, \varrho) t^{m} d \varrho \\
& =t^{-j} l_{-j}(x, \xi)+m t^{-j} \log t \frac{i}{2 \pi} \int_{\mathscr{C}(x, \xi)} q_{-m-j}(x, \xi, \varrho) d \varrho,
\end{aligned}
$$

where the last term is zero since $q_{-m-j}$ is $O\left(|\varrho|^{-2}\right)$ for $|\varrho| \rightarrow \infty$ when $j>0$. 
When $m$ is even, we see that the transmission condition (2.2) carries over through the calculations (2.10) to the corresponding property for $l(x, \xi)$, since the parity of $-j$ is the same as that of $-j-m$.

Now consider the contribution from $G_{\lambda}$. Here we shall use the following observations:

$$
\begin{aligned}
& Q_{\lambda}+\lambda^{-1}=Q_{\lambda}+\lambda^{-1}(P-\lambda) Q_{\lambda}=\lambda^{-1} P Q_{\lambda} \quad \text { on } \tilde{X}, \\
& R_{\lambda}+\lambda^{-1} \\
& \quad=R_{\lambda}+\lambda^{-1}\left(P_{+}+G-\lambda\right) R_{\lambda}=\lambda^{-1}\left(P_{+}+G\right)\left(Q_{\lambda,+}+G_{\lambda}\right) \\
& \quad=\lambda^{-1}\left[\left(P Q_{\lambda}\right)_{+}-L\left(P, Q_{\lambda}\right)+G Q_{\lambda,+}+\left(P_{+}+G\right) G_{\lambda}\right] \\
& \quad=Q_{\lambda,+}+\lambda^{-1}+\lambda^{-1}\left[-L\left(P, Q_{\lambda}\right)+G Q_{\lambda,+}+\left(P_{+}+G\right) G_{\lambda}\right] \quad \text { on } X ;
\end{aligned}
$$

they imply in view of (2.4) that $G_{\lambda}$ may be written as

$$
G_{\lambda}=\lambda^{-1}\left[-L\left(P, Q_{\lambda}\right)+G Q_{\lambda,+}+\left(P_{+}+G\right) G_{\lambda}\right] .
$$

Here $L\left(P, Q_{\lambda}\right)=G^{+}(P) G^{-}\left(Q_{\lambda}\right)$ in local coordinates. (The latter formula is accounted for in $\left[6,(1.2 .49-50)\right.$ and Sect. 2.6]; we recall that $G^{+}(P)=$ $r^{+} P e^{-} J$ and $G^{-}(P)=J r^{-} P e^{+}$, where $e^{ \pm}$extends by zero from $\mathrm{R}_{ \pm}^{n}$ to $\mathrm{R}^{n}, r^{ \pm}$ restricts from $\mathrm{R}^{n}$ to $\mathrm{R}_{ \pm}^{n}$, and $J$ is the reflection map $J: u\left(x^{\prime}, x_{n}\right) \mapsto u\left(x^{\prime},-x_{n}\right)$.) By [6, Th. 3.3.2], $G_{\lambda}$ is of order $-m$ and regularity $\nu$; moreover, (2.12) shows that it is $\lambda^{-1}$ times an s.g.o. of order 0 and regularity $v$ (by the composition rules in [6, Th. 2.7.6-7]).

Since

$$
\begin{aligned}
& Q_{\lambda}: L_{2}(\tilde{X}, \widetilde{E}) \rightarrow H^{m-\varepsilon}(\tilde{X}, \widetilde{E}), \quad \text { with norms } O\left(\lambda^{-\varepsilon / m}\right), \\
& G_{\lambda}: L_{2}(X, E) \rightarrow H^{m-\varepsilon}(X, E),
\end{aligned}
$$

for $\varepsilon \in[0, m]$ (a standard observation used also in [6, pp. 409-410]), each of the terms in [ ] in (2.12) maps $L_{2}(X, E)$ to $H^{-\varepsilon}(X, E)$ with norm $O\left(\lambda^{-\varepsilon / m}\right)$. Then we can perform the integration in this operator norm (letting $s \rightarrow 0$ ), defining the s.g.o.-like part $G^{\log }$ of $\log (P+G)_{T}$ by

$$
\begin{aligned}
G^{\log } & =\frac{i}{2 \pi} \int_{\mathscr{C}} \log \lambda G_{\lambda} d \lambda \\
& =\frac{i}{2 \pi} \int_{\mathscr{C}} \lambda^{-1} \log \lambda\left[-L\left(P, Q_{\lambda}\right)+G Q_{\lambda,+}+\left(P_{+}+G\right) G_{\lambda}\right] d \lambda
\end{aligned}
$$

also written as 


$$
\begin{aligned}
G^{\log } & =-G^{+}(P) \frac{i}{2 \pi} \int_{\mathscr{C}} \lambda^{-1} \log \lambda G^{-}\left(Q_{\lambda}\right) d \lambda \\
& +G \frac{i}{2 \pi} \int_{\mathscr{C}} \lambda^{-1} \log \lambda Q_{\lambda,+} d \lambda+\left(P_{+}+G\right) \frac{i}{2 \pi} \int_{\mathscr{C}} \lambda^{-1} \log \lambda G_{\lambda} d \lambda
\end{aligned}
$$

when localized. It is a bounded operator from $L_{2}(X, E)$ to $H^{-\varepsilon}(X, E)$. Summing up, we have found:

THEOREM 2.2. The logarithm of the realization $B=(P+G)_{T}$ satisfies

$$
\log B=\log (P+G)_{T}=(\log P)_{+}+G^{\log },
$$

where $\log P$ is the logarithm of $P$ on $\tilde{X}$, and $G^{\log }$ is defined by (2.13), (2.14); the terms are bounded operators from $L_{2}(X, E)$ to $H^{-\varepsilon}(X, E)($ any $\varepsilon>0)$.

The operator $G^{\log }$ is a generalized singular Green operator, in the same spirit as the generalized s.g.o.s $G^{(-s)}$ studied in [6, Sect. 4.4] (the s.g.o.-like parts of the powers $B^{-s}$ ), and one can show as in [6, Th. 4.4.4] that there is a symbol-kernel satisfying part of the usual $L_{2, x_{n}, y_{n}}\left(\mathbf{R}_{++}^{2}\right)$ estimates for s.g.o.s, allowing $D_{x^{\prime}}^{\beta}, D_{\xi^{\prime}}^{\alpha},\left(x_{n} D_{x_{n}}\right)^{k}$ and $\left(y_{n} D_{y_{n}}\right)^{l}$ in arbitrarily high powers (with exceptions for the principal term), and allowing some applications of $x_{n}^{k} D_{x_{n}}^{k^{\prime}}$ and $y_{n}^{l} D_{y_{n}}^{l^{\prime}}$, limited by the regularity and other restrictions. We account for this in Theorem 2.6 below; let us first consider an example.

Example 2.3. Let $P=1-\Delta$ on $\mathrm{R}_{+}^{n}$. It is easy to see that the solution operator for the Dirichlet problem for $P-\lambda=1-\Delta-\lambda, \lambda \in V=\mathrm{C} \backslash \mathrm{R}_{+}$, is $R_{\lambda}=Q_{\lambda,+}+G_{\lambda}$, where $Q_{\lambda}$ is the $\psi \operatorname{do}(1-\lambda-\Delta)^{-1}$ with symbol $\left(\langle\xi\rangle^{2}-\lambda\right)^{-1}$, and $G_{\lambda}$ is the singular Green operator with symbol-kernel $\frac{-1}{2 \kappa_{1}} e^{-\kappa_{1}\left(x_{n}+y_{n}\right)} ; \kappa_{1}=$ $\left(\left\langle\xi^{\prime}\right\rangle^{2}-\lambda\right)^{\frac{1}{2}}$. (We here use the well-known notation $\langle x\rangle=\left(x_{1}^{2}+\cdots+x_{n}^{2}+1\right)^{\frac{1}{2}}$.) It follows that

$$
\log P=\mathrm{OP}(2 \log \langle\xi\rangle)
$$

To find out how $G^{\log }$ acts on functions $\varphi \in C_{0}^{\infty}\left(\mathrm{R}_{+}^{n}\right)$, we write (using that $e^{-\kappa_{1}\left(x_{n}+y_{n}\right)}$ is rapidly decreasing in $\lambda$ on the rays in $V$ when $y_{n}$ is in the support of $\varphi$ ):

$$
\begin{aligned}
G^{\log \varphi} & =\frac{i}{2 \pi} \int_{\mathscr{C}} \log \lambda G_{\lambda} \varphi d \lambda \\
& =\frac{i}{2 \pi} \int_{\mathscr{C}} \int_{\mathrm{R}^{n-1}} \int_{0}^{\infty} \log \lambda e^{i x^{\prime} \cdot \xi^{\prime}} \frac{-1}{2 \kappa_{1}} e^{-\kappa_{1}\left(x_{n}+y_{n}\right)} \dot{\varphi}\left(\xi^{\prime}, y_{n}\right) d y_{n} d \xi^{\prime} d \lambda
\end{aligned}
$$


with $\dot{\varphi}$ denoting the partial Fourier transform $\varphi^{\prime}\left(\xi^{\prime}, y_{n}\right)=\mathscr{F}_{y^{\prime} \rightarrow \xi^{\prime}} \varphi\left(y^{\prime}, y_{n}\right)$. Here we can calculate

$$
\begin{aligned}
\frac{i}{2 \pi} \int_{\mathscr{C}} \log \lambda \frac{-1}{2 \kappa_{1}} e^{-\kappa_{1}\left(x_{n}+y_{n}\right)} d \lambda & =\int_{-\infty}^{0} \frac{1}{2\left(\left\langle\xi^{\prime}\right\rangle^{2}-t\right)^{\frac{1}{2}}} e^{-\left(\left\langle\xi^{\prime}\right\rangle^{2}-t\right)^{\frac{1}{2}}\left(x_{n}+y_{n}\right)} d t \\
& =\int_{0}^{\infty} \frac{1}{2\left(\left\langle\xi^{\prime}\right\rangle^{2}+s\right)^{\frac{1}{2}}} e^{-\left(\left\langle\xi^{\prime}\right\rangle^{2}+s\right)^{\frac{1}{2}}\left(x_{n}+y_{n}\right)} d s \\
& =\int_{\left\langle\xi^{\prime}\right\rangle}^{\infty} \frac{1}{2 u} e^{-u\left(x_{n}+y_{n}\right)} 2 u d u \\
& =\frac{1}{x_{n}+y_{n}} e^{-\left\langle\xi^{\prime}\right\rangle\left(x_{n}+y_{n}\right)},
\end{aligned}
$$

using that the $\log |\lambda|$ contributions cancel out (as in [8, Lemma 1.2]). Thus

$$
G^{\log } \varphi=\int_{\mathrm{R}^{n-1}} \int_{0}^{\infty} e^{i x^{\prime} \cdot \xi^{\prime}} \frac{1}{x_{n}+y_{n}} e^{-\left\langle\xi^{\prime}\right\rangle\left(x_{n}+y_{n}\right)} \dot{\varphi}\left(\xi^{\prime}, y_{n}\right) d y_{n} d \xi^{\prime} .
$$

This shows that $G^{\log }$ is a generalized kind of s.g.o. with symbol-kernel

$$
\tilde{g}^{\log }\left(x^{\prime}, x_{n}, y_{n}, \xi^{\prime}\right)=\frac{1}{x_{n}+y_{n}} e^{-\left\langle\xi^{\prime}\right\rangle\left(x_{n}+y_{n}\right)} .
$$

Since the operator with kernel $\frac{1}{x_{n}+y_{n}}$ is bounded in $L_{2}\left(R_{+}\right)$(as a truncation of the Hilbert transform), it follows that $G^{\log }$ is a bounded operator in $L_{2}\left(\mathrm{R}_{+}^{n}\right)$.

Note that $\partial_{\xi_{1}} \tilde{g}^{\log }$ is a standard s.g.o. symbol-kernel, and that $x_{n} \tilde{g}^{\log }$ is bounded.

The same calculations with $\left\langle\xi^{\prime}\right\rangle$ replaced by $\left|\xi^{\prime}\right|$ show that for $P=-\Delta$, $G^{\log }$ has symbol-kernel $\frac{1}{x_{n}+y_{n}} e^{-\left|\xi^{\prime}\right|\left(x_{n}+y_{n}\right)}$ for $\left|\xi^{\prime}\right| \geq 1$.

In the general differential operator case, $G^{\log }$ is qualitatively very much like in this example. Here one can directly use the symbol-kernel estimates and boundedness considerations worked out by Seeley in [17], [18]. Notationally, we follow [8]; in particular, the enumeration of quasi-homogeneous (resp. homogeneous) terms in the asymptotic expansions of singular Green symbolkernels (resp. symbols) have been shifted by one step in comparison with [6], in order to have the same index on an s.g.o. symbol-kernel (resp. symbol) and its normal trace. For example, the principal part of a symbol-kernel $\tilde{g}$ of order $-m$ is denoted $\tilde{g}_{-m}$ (although the corresponding symbol $g_{-m}$ has homogeneity degree $-m-1)$. We shall use the notation $\dot{\leq}$ (resp. $\dot{\geq}$ ) to indicate "less than or equal (resp. greater than or equal) to a constant times", and $\doteq$ to indicate that both $\dot{\leq}$ and $\dot{\geq}$ hold. 
Theorem 2.4. Consider the case where $P$ is a differential operator, $G=0$, and the trace operators $T_{0}, \ldots, T_{m-1}$ are differential operators. In this case, the singular Green part $G_{\lambda}$ of the resolvent is of regularity $+\infty$ and its symbolkernel in local coordinates $\tilde{g} \sim \sum_{j \geq 0} \tilde{g}_{-m-j}$, expanded in quasi-homogeneous terms

$$
\begin{aligned}
& \tilde{g}_{-m-j}\left(x^{\prime}, \frac{x_{n}}{t}, \frac{y_{n}}{t}, t \xi^{\prime}, t^{m} \lambda\right) \\
& \quad=t^{-m+1-j} \tilde{g}_{-m-j}\left(x^{\prime}, x_{n}, y_{n}, \xi^{\prime}, \lambda\right) \quad \text { for } t \geq 1,\left|\xi^{\prime}\right| \geq 1
\end{aligned}
$$

satisfies estimates on the rays in $V$, with $\kappa=\left|\xi^{\prime}\right|+|\lambda|^{\frac{1}{m}}$ :

$$
\left|D_{x^{\prime}}^{\beta} D_{\xi^{\prime}}^{\alpha} x_{n}^{k} D_{x_{n}}^{k^{\prime}} y_{n}^{l} D_{y_{n}}^{l^{\prime}} D_{\lambda}^{p} \tilde{g}_{-m-j}\right| \dot{\leq} \kappa^{1-m-|\alpha|-k+k^{\prime}-l+l^{\prime}-j-m p} e^{-c \kappa\left(x_{n}+y_{n}\right)}
$$

for all indices, when $\kappa \geq \varepsilon$.

Then $G^{\log }$ is, in local coordinates near $X^{\prime}$, a generalized singular Green operator

$$
\begin{aligned}
G^{\log } u(x) & =\int_{\mathrm{R}^{n-1}} \int_{0}^{\infty} e^{i x^{\prime} \cdot \xi^{\prime}} \tilde{g}^{\log }\left(x^{\prime}, x_{n}, y_{n}, \xi^{\prime}\right) \dot{u}\left(\xi^{\prime}, y_{n}\right) d y_{n} d \xi^{\prime} \\
& =\operatorname{OPG}\left(\tilde{g}^{\log }\left(x^{\prime}, x_{n}, y_{n}, \xi^{\prime}\right)\right) u(x)
\end{aligned}
$$

with $\tilde{g}^{\log } \sim \sum_{j \in \mathrm{N}} \tilde{g}_{-j}^{\log }$; here the $j$ 'th term is quasihomogeneous:

$$
\begin{aligned}
& \tilde{g}_{-j}^{\log }\left(x^{\prime}, \frac{x_{n}}{t}, \frac{y_{n}}{t}, t \xi^{\prime}\right) \\
& =t^{1-j} \tilde{g}_{-j}^{\log }\left(x^{\prime}, x_{n}, y_{n}, \xi^{\prime}\right) \quad \text { for } t \geq 1 \text { and }\left|\xi^{\prime}\right| \geq 1
\end{aligned}
$$

and satisfies, when $\left|\xi^{\prime}\right| \geq \varepsilon$,

$$
\left|D_{x^{\prime}}^{\beta} D_{\xi^{\prime}}^{\alpha} x_{n}^{k} D_{x_{n}}^{k^{\prime}} y_{n}^{l} D_{y_{n}}^{l^{\prime}} \tilde{g}_{-j}^{\log }\right| \dot{\leq}\left|\xi^{\prime}\right|^{-|\alpha|-k+k^{\prime}-l+l^{\prime}-j} \frac{1}{x_{n}+y_{n}} e^{-c\left|\xi^{\prime}\right|\left(x_{n}+y_{n}\right)}
$$

for the indices satisfying

$$
-k+k^{\prime}-l+l^{\prime}-|\alpha|-j \leq 0 .
$$

It follows in particular that $G^{\log }$ is a bounded operator in $L_{p}(X, E)$ for $1<p<\infty$.

Proof. The estimates (2.20) were shown in [17, (29)], [18]. Because of the fall-off in $\lambda$, they allow us to define the $j$ 'th term in the symbol-kernel of $G^{\log }$ 
for $\left|\xi^{\prime}\right| \geq \varepsilon$ by

$$
\begin{aligned}
\tilde{g}_{-j}^{\log }\left(x^{\prime}, x_{n}, y_{n}, \xi^{\prime}\right) & =\frac{i}{2 \pi} \int_{\mathscr{C}} \log \lambda \tilde{g}_{-m-j}\left(x^{\prime}, x_{n}, y_{n}, \xi^{\prime}, \lambda\right) d \lambda \\
& =\int_{0}^{\infty} \tilde{g}_{-m-j}\left(x^{\prime}, x_{n}, y_{n}, \xi^{\prime},-s\right) d s ;
\end{aligned}
$$

here we rewrote the integral as in (2.17) (and [8, Lemma 1.2]). The homogeneity is seen from the last integral, using (2.19). The function is estimated as follows, for the indices satisfying (2.24), when we use that $\left|\xi^{\prime}\right|+s^{\frac{1}{m}} \doteq$ $\left(\left|\xi^{\prime}\right|^{m}+s\right)^{\frac{1}{m}}$ :

$$
\begin{aligned}
\mid D_{x^{\prime}}^{\beta} & D_{\xi^{\prime}}^{\alpha} x_{n}^{k} D_{x_{n}}^{k^{\prime}} y_{n}^{l} D_{y_{n}}^{l^{\prime}} \tilde{g}_{-j}^{\log } \mid \\
& =\left|\int_{0}^{\infty} D_{x^{\prime}}^{\beta} D_{\xi^{\prime}}^{\alpha} x_{n}^{k} D_{x_{n}}^{k^{\prime}} y_{n}^{l} D_{y_{n}}^{l^{\prime}} \tilde{g}_{-m-j}\left(x^{\prime}, x_{n}, y_{n}, \xi^{\prime},-s\right) d s\right| \\
& \leq\left|\xi^{\prime}\right|^{-|\alpha|-k+k^{\prime}-l+l^{\prime}-j} \int_{0}^{\infty}\left(\left(\left|\xi^{\prime}\right|^{m}+s\right)^{\frac{1}{m}}\right)^{1-m} e^{-c\left(\xi^{\prime}||^{m}+s\right)^{\frac{1}{m}}\left(x_{n}+y_{n}\right)} d s \\
& =\left|\xi^{\prime}\right|^{-|\alpha|-k+k^{\prime}-l+l^{\prime}-j} \int_{\left|\xi^{\prime}\right|}^{\infty} u^{1-m} e^{-c u\left(x_{n}+y_{n}\right)} m u^{m-1} d u \\
& =\left|\xi^{\prime}\right|^{-|\alpha|-k+k^{\prime}-l+l^{\prime}-j} \frac{m}{c\left(x_{n}+y_{n}\right)} e^{-c\left|\xi^{\prime}\right|\left(x_{n}+y_{n}\right)} .
\end{aligned}
$$

The operator $G^{\log }$ is defined from a finite number of these symbol terms multiplied with an excision function $\zeta\left(\left|\xi^{\prime}\right|\right)$, where

$$
\zeta(t) \in C^{\infty}(\mathrm{R}), \quad \zeta(t)=0 \text { for }|t| \leq \delta_{1}, \quad \zeta(t)=1 \text { for }|t| \geq \delta_{2},
$$

plus an integral as in (2.13) of the remainder of $G_{\lambda}$, which can be taken with arbitrarily high smoothness of the kernel and decrease for $\lambda \rightarrow \infty$, cf. [18, (2.14)]. Applying the arguments of Theorem 1 of [18] (using Lemmas 1 and 2 there invoking Mihlin's theorem and the Hilbert transform) one finds that $G^{\text {log }}$ is $L_{p}$-continuous as asserted.

Remark 2.5. The lower order terms in $\tilde{g}^{\log }$ and the derivatives are not as singular for $x_{n}+y_{n} \rightarrow 0$ as (2.23) indicates. In fact, the symbol-kernels one step down can be estimated as follows:

$$
\text { When }-k+k^{\prime}-l+l^{\prime}-|\alpha|-j \leq-1 \text {, }
$$




$$
\begin{aligned}
\left|D_{x^{\prime}}^{\beta} D_{\xi^{\prime}}^{\alpha} x_{n}^{k} D_{x_{n}}^{k^{\prime}} y_{n}^{l} D_{y_{n}}^{l^{\prime}} \tilde{g}_{-j}^{\log \mid}\right| & \dot{\leq}\left|\xi^{\prime}\right|^{-|\alpha|-k+k^{\prime}-l+l^{\prime}-j+1} \int_{\left|\xi^{\prime}\right|}^{\infty} u^{-1-\varepsilon} u^{\varepsilon} e^{-c u\left(x_{n}+y_{n}\right)} d u \\
& \dot{\leq}\left|\xi^{\prime}\right|^{-|\alpha|-k+k^{\prime}-l+l^{\prime}-j+1+\varepsilon} \sup _{u \in \mathrm{R}_{+}}\left|u^{\varepsilon} e^{-c u\left(x_{n}+y_{n}\right)}\right| \\
& \dot{\leq}\left|\xi^{\prime}\right|^{-|\alpha|-k+k^{\prime}-l+l^{\prime}-j+1+\varepsilon}\left(x_{n}+y_{n}\right)^{-\varepsilon},
\end{aligned}
$$

for $\varepsilon>0$. The symbol-kernels two steps down are bounded for $x_{n}+y_{n} \rightarrow 0$ :

$$
\text { When }-k+k^{\prime}-l+l^{\prime}-|\alpha|-j \leq-2 \text {, }
$$

$$
\begin{aligned}
\left|D_{x^{\prime}}^{\beta} D_{\xi^{\prime}}^{\alpha} x_{n}^{k} D_{x_{n}}^{k^{\prime}} y_{n}^{l} D_{y_{n}}^{l^{\prime}} \tilde{g}_{-j}^{\log \mid}\right| & \dot{\leq}\left|\xi^{\prime}\right|^{-|\alpha|-k+k^{\prime}-l+l^{\prime}-j+2} \int_{0}^{\infty}\left(\left|\xi^{\prime}\right|+s^{\frac{1}{m}}\right)^{-m-1} d s \\
& \dot{\leq}\left|\xi^{\prime}\right|^{-|\alpha|-k+k^{\prime}-l+l^{\prime}-j+1},
\end{aligned}
$$

and the smoothness at 0 increases with increasing $|\alpha|$ and $j$.

Now let us turn to the pseudodifferential case and the methods of $[6$, Sect. 4.4].

THEOREM 2.6. Let $\left\{P_{+}+G, T\right\}$ have regularity $v \in\left[\frac{1}{2}, \infty\left[\right.\right.$, and define $G^{\log }$ by (2.13). Then $G^{\log }$ is, in local coordinates near $X^{\prime}$, a generalized singular Green operator as in (2.21) with $\tilde{g}^{\log } \sim \sum_{j \in \mathrm{N}} \tilde{g}_{-j}^{\log }$; here the $j$ 'th term is quasihomogeneous as in (2.22) when $j>0$, and the series approximates $\tilde{g}^{\log }$ asymptotically in the sense that

$$
\left\|D_{x^{\prime}}^{\beta} D_{\xi^{\prime}}^{\alpha} x_{n}^{k} D_{x_{n}}^{k^{\prime}} y_{n}^{l} D_{y_{n}}^{l^{\prime}}\left[\tilde{g}^{\log }-\sum_{j<J} \tilde{g}_{-j}^{\log }\right]\right\|_{L_{2, x_{n}, y_{n}}} \dot{\leq}\left\langle\xi^{\prime}\right\rangle^{-|\alpha|-k+k^{\prime}-l+l^{\prime}-J}
$$

holds for the indices satisfying

$$
\begin{array}{r}
-k+k^{\prime}-l+l^{\prime}-|\alpha|-J<0, \\
{\left[k-k^{\prime}\right]_{-}+\left[l-l^{\prime}\right]_{-}<v .}
\end{array}
$$

Moreover,

$$
\left\|D_{x^{\prime}}^{\beta} D_{\xi^{\prime}}^{\alpha} x_{n}^{k} D_{x_{n}}^{k^{\prime}} y_{n}^{l} D_{y_{n}}^{l^{\prime}} \tilde{g}_{-J}^{\log }\right\|_{L_{2, x_{n}, y_{n}}} \dot{\leq}\left\langle\xi^{\prime}\right\rangle^{-|\alpha|-k+k^{\prime}-l+l^{\prime}-J}
$$

holds for these indices.

With $\zeta(t)$ defined as in (2.27), the above symbol-kernels multiplied with $\zeta\left(x_{n}\right) \zeta\left(y_{n}\right)$ satisfy estimates for all $\alpha, \beta, J, k, k^{\prime}, l, l^{\prime}$ with $\left\langle\xi^{\prime}\right\rangle^{-M}$, any $M$, in the right-hand side.

Proof. This is modeled after the proof of [6, Th. 4.4.4] and the remarks preceding it. 
We recall from [6, Th. 3.3.9] that the symbol-kernel $\tilde{g}\left(x^{\prime}, x_{n}, y_{n}, \xi^{\prime}, \lambda\right)$ of $G_{\lambda}$ (in a local coordinate system) has an expansion in quasi-homogeneous terms $\tilde{g} \sim \sum_{j \geq 0} \tilde{g}_{-m-j}$ satisfying (2.19) in $V$, and that one has for all indices, denoting $\lambda=-\mu^{m} e^{i \omega}(\mu>0),\left(\left|\xi^{\prime}\right|^{2}+\mu^{2}+1\right)^{\frac{1}{2}}=\left\langle\xi^{\prime}, \mu\right\rangle$ :

$$
\begin{aligned}
& \left\|D_{x^{\prime}, \omega}^{\beta} D_{\xi^{\prime}}^{\alpha} x_{n}^{k} D_{x_{n}}^{k^{\prime}} y_{n}^{l} D_{y_{n}}^{l^{\prime}}\left[\tilde{g}-\sum_{j<J} \tilde{g}_{-m-j}\right]\right\|_{L_{2, x_{n}, y_{n}}} \\
& \dot{\leq}\left(\left\langle\xi^{\prime}\right\rangle^{\nu-M^{\prime}}+\left\langle\xi^{\prime}, \mu\right\rangle^{\nu-M^{\prime}}\right)\left\langle\xi^{\prime}, \mu\right\rangle^{-m-\nu+M^{\prime \prime}} \\
& \dot{\leq} \begin{cases}\left\langle\xi^{\prime}, \mu\right\rangle^{-m-M^{\prime}+M^{\prime \prime},}, & \text { when } M^{\prime} \leq v, \\
\left\langle\xi^{\prime}\right\rangle^{\nu-M^{\prime}}\left\langle\xi^{\prime}, \mu\right\rangle^{-m-v+M^{\prime \prime}}, & \text { when } M^{\prime} \geq v,\end{cases}
\end{aligned}
$$

with

$$
\begin{aligned}
M^{\prime} & =\left[k-k^{\prime}\right]_{+}+\left[l-l^{\prime}\right]_{+}+|\alpha|+J, \\
M^{\prime \prime} & =\left[k-k^{\prime}\right]_{-}+\left[l-l^{\prime}\right]_{-} ; \quad \text { so } \\
-M^{\prime}+M^{\prime \prime} & =-k+k^{\prime}-l+l^{\prime}-|\alpha|-J .
\end{aligned}
$$

The notation $N_{ \pm}=\max \{ \pm N, 0\}$ is used, and we have (as recalled earlier) changed the indexation from [6] by one step as in [8].

Let us first observe that the "error terms" and remainders in the resolvent construction, that are negligible in the class of operators of order $-m$ and regularity $v$, give rise to generalized s.g.o. error terms $G^{\prime}$ here, satisfying estimates of the type (as in [6, Lemma 2.3.11])

$$
\begin{aligned}
\| D_{x^{\prime}}^{\beta} & D_{\xi^{\prime}}^{\alpha} x_{n}^{k} D_{x_{n}}^{k^{\prime}} y_{n}^{l} D_{y_{n}}^{l^{\prime}} \tilde{g}^{\prime} \|_{L_{2, x_{n}, y_{n}}} \\
& \leq\left\langle\xi^{\prime}\right\rangle^{-M}\left|\int_{\mathscr{C}} \log \lambda\langle\lambda\rangle^{-1-\left(\nu-\left[k-k^{\prime}\right]_{-}-\left[l-l^{\prime}\right]_{-}\right) / m} d \lambda\right| \\
& \leq\left\langle\xi^{\prime}\right\rangle^{-M}, \quad \text { for any } M \text {, when } \quad\left[k-k^{\prime}\right]_{-}+\left[l-l^{\prime}\right]_{-}<v .
\end{aligned}
$$

It follows that the corresponding kernels $\mathscr{K}_{G^{\prime}}(x, y)$ satisfy, for these indices:

$$
\sup _{x^{\prime}, y^{\prime}}\left\|D_{x^{\prime}, y^{\prime}}^{\gamma} x_{n}^{k} D_{x_{n}}^{k^{\prime}} y_{n}^{l} D_{y_{n}}^{l^{\prime}} \mathscr{K}_{G^{\prime}}\right\|_{L_{2, x_{n}, y_{n}}}<\infty
$$

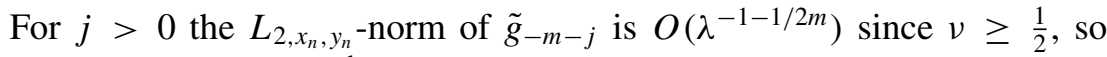
the corresponding term $\tilde{g}_{-j}^{\log }$ can be defined directly for $\left|\xi^{\prime}\right| \geq 1$ by Cauchy integrals as in (2.25), convergent in the $L_{2, x_{n}, y_{n}}$-norm. The quasi-homogeneity of $\tilde{g}_{-j}^{\log }$ is seen as in (2.25) by using [8, Lemma 1.2] in $L_{2, x_{n}, y_{n}}$-norm. 
We use the estimates (2.33) to see that for $\tilde{g}^{\log }-\sum_{j<J} \tilde{g}_{-j}^{\log }$ with $J>0$ (so that the first term is excluded), the integrand in the corresponding Cauchy integral is $O\left(\lambda^{-1-\varepsilon}\right)$ in $L_{2, x_{n}, y_{n}}$-norm (some $\varepsilon>0$ ), when

$$
\text { (2.37) }-k+k^{\prime}-l+l^{\prime}-|\alpha|-J<0 \text {, if }\left[k-k^{\prime}\right]_{+}+\left[l-l^{\prime}\right]_{+}+|\alpha|+J \leq v,
$$

and when

$$
\left[k-k^{\prime}\right]_{-}+\left[l-l^{\prime}\right]_{-}<v, \text { if }\left[k-k^{\prime}\right]_{+}+\left[l-l^{\prime}\right]_{+}+|\alpha|+J \geq v .
$$

Then the integral converges and defines a symbol-kernel satisfying the asserted estimate. Since

$$
-k+k^{\prime}-l+l^{\prime}-|\alpha|-J=\left[k-k^{\prime}\right]_{-}+\left[l-l^{\prime}\right]_{-}-\left(\left[k-k^{\prime}\right]_{+}+\left[l-l^{\prime}\right]_{+}+|\alpha|+J\right),
$$

we see that the conditions "if ..." can be left out in (2.37)-(2.38), leading to the formulation (2.31).

We still have to consider the first term $\tilde{g}_{0}^{\log }$ in $\tilde{g}^{\log }$, defined from the principal part $\tilde{g}_{-m}$ of $\tilde{g}$. Here we use that $\tilde{g}_{-m}$ can be found by performing the resolvent construction on the principal boundary symbol level for the corresponding operators on $L_{2}\left(\mathbf{R}_{+}\right)$, and that they obey a one-dimensional version of the identities in (2.11). So we can replace $\tilde{g}_{-m}$ by the symbol-kernel of the principal boundary symbol version of (2.12), which gives a convergent Cauchy integral, when the $\lambda$-independent factors are pulled outside of the integration. In a formal sense, we can ascribe it a symbol-kernel $\tilde{g}_{0}^{\log }\left(x^{\prime}, x_{n}, y_{n}, \xi^{\prime}\right)$. The resulting boundary symbol operator is continuous from $L_{2}\left(\mathbf{R}_{+}\right)$to $H^{-\varepsilon}\left(\mathbf{R}_{+}\right)$ for $\varepsilon>0$, at each $\left(x^{\prime}, \xi^{\prime}\right)$. If we define the functions derived from $\tilde{g}_{0}^{\log }$ "weakly" by

$$
\begin{aligned}
D_{x^{\prime}}^{\beta} D_{\xi^{\prime}}^{\alpha} x_{n}^{k} D_{x_{n}}^{k^{\prime}} y_{n}^{l} D_{y_{n}}^{l^{\prime}} \tilde{g}_{0}^{\log }\left(x^{\prime}, x_{n}, y_{n}, \xi^{\prime}\right) \\
\quad=\frac{i}{2 \pi} \int_{\mathscr{C}} \log \lambda D_{x^{\prime}}^{\beta} D_{\xi^{\prime}}^{\alpha} x_{n}^{k} D_{x_{n}}^{k^{\prime}} y_{n}^{l} D_{y_{n}}^{l^{\prime}} \tilde{g}_{-m}\left(x^{\prime}, x_{n}, y_{n}, \xi^{\prime}, \lambda\right) d \lambda
\end{aligned}
$$

we can use that the integral converges in $L_{2, x_{n}, y_{n}}$-norm when the indices satisfy (2.31). In this sense, the estimates (2.30) hold also when $J=0$ in (2.31).

The estimates (2.32) of the individual terms follow from (2.30) since $\tilde{g}_{-J}^{\log }=$ $\left(\tilde{g}^{\log }-\sum_{j<J} \tilde{g}_{-j}^{\log }\right)-\left(\tilde{g}^{\log }-\sum_{j<J+1} \tilde{g}_{-j}^{\log }\right)$.

Finally, for the statements on the symbol-kernels multiplied with $\zeta\left(x_{n}\right) \zeta\left(y_{n}\right)$, note that $\zeta(t)$ can for any $k \in \mathrm{N}$ be written as $t^{k} \zeta_{k}(t)$ with a bounded smooth function $\zeta_{k}$, so from the already shown estimates we can infer arbitrarily rapid fall-off in $\xi^{\prime}$ by rewriting with arbitrarily high powers of $x_{n}$ and $y_{n}$. 
If $R_{\lambda}$ has infinite regularity, $v$ can be arbitrarily large in the second line of (2.31), so the line can be left out. Note that even then there is a limitation on the indices for which we get standard s.g.o. estimates.

While $G^{\log }$ is the primary s.g.o.-type operator to consider in this connection, it is also of interest to study some other s.g.o.-type operators here, namely, in local coordinates, $G^{+}(\log P)=r^{+}(\log P) e^{-} J$ and $G^{-}(\log P)=$ $J r^{-}(\log P) e^{+}$, with notation as in the text after (2.12). The operators $G^{ \pm}(\log P)$ have properties very similar to those of $G^{\log }$ :

TheOREM 2.7. The operators $G^{ \pm}(\log P)$ are defined in local coordinates by

$$
\begin{aligned}
G^{+}(\log P) & =r^{+} \log P e^{-} J=r^{+} \frac{i}{2 \pi} \int_{\mathscr{C}} \log \lambda Q_{\lambda} d \lambda e^{-} J \\
& =\frac{i}{2 \pi} \int_{\mathscr{C}} \lambda^{-1} \log \lambda G^{+}\left(P Q_{\lambda}\right) d \lambda, \\
G^{-}(\log P) & =J r^{-} \log P e^{+}=J r^{-} \frac{i}{2 \pi} \int_{\mathscr{C}} \log \lambda Q_{\lambda} d \lambda e^{+} \\
& =\frac{i}{2 \pi} \int_{\mathscr{C}} \lambda^{-1} \log \lambda G^{-}\left(P Q_{\lambda}\right) d \lambda .
\end{aligned}
$$

Their symbol-kernels $\tilde{g}^{ \pm}(\log p)$ have properties like those of $\tilde{g}^{\log }$ in Theorem 2.6, with $v=m$.

In particular, when $P$ is a differential operator, the s.g.o.s $G^{ \pm}\left(Q_{\lambda}\right)$ satisfy Seeley's estimates (2.20), and hence the operators $G^{ \pm}(\log P)$ have symbol estimates and boundedness properties like those of $G^{\log }$ in Theorem 2.4, Remark 2.5.

Proof. The defining integrals are established by use of the first formula in (2.11), noting that $G^{ \pm}\left(\lambda^{-1}\right)=0$. By [6, Th. 2.7.4], $G^{ \pm}\left(Q_{\lambda}\right)$ is a parameterdependent polyhomogeneous family of s.g.o.s of order $-m$ and regularity $m-\varepsilon$ (any $\varepsilon>0$ ), since $Q_{\lambda}$ is of order $-m$ and regularity $m$. The symbol-kernel then satisfies estimates like those for $\tilde{g}$ in Theorem 2.6, with $v=m-\varepsilon$. The method of Theorem 2.6 leads to the conclusion that the resulting symbol-kernel $\tilde{g}^{ \pm}(\log p)$ has properties like those stated for $\tilde{g}^{\log }$, with $v=m-\varepsilon$; here $\varepsilon$ can be removed since the second inequality in (2.31) is sharp.

For the second statement, we must show that the Seeley estimates (2.20) are valid for the homogeneous terms in the symbol-kernel of $G^{ \pm}\left(Q_{\lambda}\right)$. But this is easy. Consider e.g. $G^{+}\left(Q_{\lambda}\right)$. Using the Taylor expansion of the symbol of $Q_{\lambda}$ at $x_{n}=0$ :

$$
q\left(x^{\prime}, x_{n}, \xi, \lambda\right) \sim \sum_{l \in \mathrm{N}} \frac{1}{l !} x_{n}^{l} \partial_{x_{n}}^{l} q\left(x^{\prime}, 0, \xi, \lambda\right)
$$


we have from [6, Th. 2.7.4] that

$$
g^{+}(q)\left(x^{\prime}, \xi, \eta_{n}, \lambda\right) \sim \sum_{l \in \mathrm{N}} \frac{1}{l !} \bar{D}_{\xi_{n}}^{l} g^{+}\left[\partial_{x_{n}}^{l} q\left(x^{\prime}, 0, \xi, \lambda\right)\right],
$$

where $g^{+}[f]\left(\xi_{n}, \eta_{n}\right)$ is the s.g.o. symbol corresponding to the symbol-kernel $\tilde{g}^{+}[f]\left(x_{n}, y_{n}\right)$ defined by:

$$
\tilde{g}^{+}[f]\left(x_{n}, y_{n}\right)=\left.\left(r_{z_{n}}^{+}\left[\mathscr{F}_{\xi_{n} \rightarrow z_{n}}^{-1} f\right]\right)\right|_{z_{n}=x_{n}+y_{n}} .
$$

The homogeneous terms in the symbols $\partial_{x_{n}}^{l} q\left(x^{\prime}, 0, \xi, \lambda\right)$ are rational functions of $\xi_{n}$ with $\frac{1}{2} m \operatorname{dim} E$ poles in $C_{ \pm}=\{z \in \mathrm{C} \mid \operatorname{Im} z \gtrless 0\}$, lying inside a circle of radius $C \kappa$ and having a distance $\geq c \kappa$ from the real axis, for suitable positive constants $C>c$. (A more detailed description is given e.g. in [6, Remark 3.3.7].) For simplicity of notation, consider the $j$ 'th term $q_{-m-j}$ itself. The inverse Fourier transform evaluated at $z_{n}>0$ can be written as an integral of $e^{i z_{n} \xi_{n}} q_{-m-j}\left(x^{\prime}, 0, \xi^{\prime}, \xi_{n}\right)$ over the curve bounding the intersection of the circle $\left\{\left|\xi_{n}\right|=C \kappa\right\}$ with the halfplane $\left\{\operatorname{Im} \xi_{n} \geq c \kappa\right\}$ (lying in $C_{+}$). We get the factor $e^{-c \kappa z_{n}}$ since $\left|e^{i z_{n} \xi_{n}}\right| \leq e^{-c \kappa z_{n}}$ on the curve. (Similarly, the inverse Fourier transform evaluated at $z_{n}<0$ can be written as an integral over a closed curve in $\mathrm{C}_{-}$with $\operatorname{Im} \xi_{n} \leq-c \kappa$.) For the resulting symbol-kernel, this gives the factor $e^{-c \kappa\left(x_{n}+y_{n}\right)}$; the power of $\kappa$ in front is seen from the degree of the rational function.

Once the estimates (2.20) are established, the rest of the proof goes as in Theorem 2.4.

Example 2.8. For $P=1-\Delta$ as in Example 2.3, one finds by direct calculation of the inverse Fourier transform w.r.t. $\xi_{n}$ that $G^{ \pm}\left(Q_{\lambda}\right)$ both have the symbol-kernel

$$
\tilde{g}^{+}=\tilde{g}^{-}=\frac{1}{2 \kappa_{1}} e^{-\kappa_{1}\left(x_{n}+y_{n}\right)},
$$

with $\kappa_{1}=\left(\left\langle\xi^{\prime}\right\rangle^{2}-\lambda\right)^{\frac{1}{2}}$. Then the calculations of Example 2.3 can be used again, to see that

$$
\tilde{g}^{+}(\log p)\left(x^{\prime}, x_{n}, y_{n}, \xi^{\prime}\right)=\tilde{g}^{-}(\log p)\left(x^{\prime}, x_{n}, y_{n}, \xi^{\prime}\right)=\frac{-1}{x_{n}+y_{n}} e^{-\left\langle\xi^{\prime}\right\rangle\left(x_{n}+y_{n}\right)} .
$$

For $P=-\Delta$, the calculations give that the symbol-kernel of $G^{ \pm}(\log P)$ is $\frac{-1}{x_{n}+y_{n}} e^{-\left|\xi^{\prime}\right|\left(x_{n}+y_{n}\right)}$ for $\left|\xi^{\prime}\right| \geq 1$; the same holds for $P=\mathrm{OP}\left([\xi]^{2}\right)$.

When the order $m$ is even, there is a remarkable simplification in view of Lemma 2.1: 
Proposition 2.9. When $m=2 k$, $k$ integer $>0$, then in local coordinates, the symbol-kernel of $G^{ \pm}(\log P)$ satisfies for $\left|\xi^{\prime}\right| \geq 1$ :

$$
\tilde{g}^{ \pm}(\log p)\left(x^{\prime}, x_{n}, y_{n}, \xi^{\prime}\right)=\frac{-k}{x_{n}+y_{n}} e^{-\left|\xi^{\prime}\right|\left(x_{n}+y_{n}\right)}+\tilde{g}^{ \pm, 0}\left(x^{\prime}, x_{n}, y_{n}, \xi^{\prime}\right),
$$

where $\tilde{g}^{ \pm, 0}\left(x^{\prime}, x_{n}, y_{n}, \xi^{\prime}\right)$ is a standard singular Green symbol of order and class 0 .

Proof. We here have in view of Lemma 2.1 that the symbol of $\log P$ is the sum of $k \log [\xi]^{2}$ and a symbol $l(x, \xi)$ of order 0 satisfying the transmission condition. Then we can apply Example 2.8 to the first term and the standard $G^{ \pm}$construction (of [6]) to the second term.

Thus in the even-order case, the terms in $G^{ \pm}(\log P)$ of order $<0$ satisfy all the standard s.g.o. estimates.

\section{Trace formulas}

The normal $\operatorname{trace} \operatorname{tr}_{n} G$ of a singular Green operator $G$ with symbol-kernel $\tilde{g}\left(x^{\prime}, x_{n}, y_{n}, \xi^{\prime}\right)$ in a local coordinate system is the $\psi \operatorname{do} S=\operatorname{tr}_{n} G$ with symbol

$$
s\left(x^{\prime}, \xi^{\prime}\right)=\left(\operatorname{tr}_{n} \tilde{g}\right)\left(x^{\prime}, \xi^{\prime}\right)=\int_{0}^{\infty} \tilde{g}\left(x^{\prime}, x_{n}, x_{n}, \xi^{\prime}\right) d x_{n} .
$$

In the differential operator case, we see from the estimates (2.23), (2.28), (2.29) that $\operatorname{tr}_{n} \tilde{g}_{-j}^{\log }$ is well-defined for $j \geq 1$. (Example 2.3 shows that this will generally not hold for the principal part.) In view of the homogeneity (2.22), $\operatorname{tr}_{n} \tilde{g}_{-j}^{\log }$ is homogeneous of degree $-j$ in $\xi^{\prime}$ for $\left|\xi^{\prime}\right| \geq 1$, hence a classical $\psi$ do symbol of degree $-j$. In the pseudodifferential case, we have when $v>1$ and $j \geq 1$ that the $L_{2, x_{n}, y_{n}}$-estimates of $\tilde{g}_{-j}^{\log }, y_{n} \tilde{g}_{-j}^{\log }, \partial_{y_{n}} \tilde{g}_{-j}^{\log }$ and $y_{n} \partial_{y_{n}} \tilde{g}_{-j}^{\log }$ imply as in [6, pf. of Th. 3.3.9] that there is a well-defined normal trace, again a homogeneous classical symbol of order $-j$. This estimation applies also to remainders $\tilde{g}^{\log }-\sum_{j<J} \tilde{g}_{-j}^{\log }$ for $J \geq 1$.

For $v=\frac{1}{2}$ or 1 , the estimates in Theorem 2.6 do not provide the estimates of $\partial_{y_{n}} \tilde{g}_{-j}^{\log }$ needed for this argument. However, it is still posssible to take the normal trace of $G_{\lambda}$, subtract the principal part, and integrate the remaining operator with $\log \lambda$ to get a classical $\psi$ do of order -1 .

THeOREM 3.1. In a local coordinate system, let $S_{\lambda}=\operatorname{tr}_{n} G_{\lambda}$ with symbol $s\left(x^{\prime}, \xi^{\prime}, \lambda\right)=\left(\operatorname{tr}_{n} \tilde{g}\right)\left(x^{\prime}, \xi^{\prime}, \lambda\right)$, expanded in terms $s_{-m-j}\left(x^{\prime}, \xi^{\prime}, \lambda\right)=$ $\left(\operatorname{tr}_{n} \tilde{g}_{-m-j}\right)\left(x^{\prime}, \xi^{\prime}, \lambda\right)$. Define the parts of $G_{\lambda}$ and $S_{\lambda}$ of order $-m-1$ by

$$
\begin{aligned}
G_{\lambda, \text { sub }} & =G_{\lambda}-\operatorname{OPG}\left(\tilde{g}_{-m}\left(x^{\prime}, x_{n}, y_{n}, \xi^{\prime}, \lambda\right)\right), \\
S_{\lambda, \text { sub }} & =\operatorname{tr}_{n} G_{\lambda, \text { sub }}=S_{\lambda}-\mathrm{OP}^{\prime}\left(s_{-m}\left(x^{\prime}, \xi^{\prime}, \lambda\right)\right)
\end{aligned}
$$


(the remainders after subtracting principal parts), and let

$$
G_{\text {sub }}^{\log }=\frac{i}{2 \pi} \int_{\mathscr{C}} \log \lambda G_{\lambda, \text { sub }} d \lambda,
$$

with symbol-kernel $\tilde{g}_{\text {sub }}^{\log }=\tilde{g}^{\log }-\tilde{g}_{0}^{\log }$. The formula

$$
S_{\mathrm{sub}}^{\log }=\frac{i}{2 \pi} \int_{\mathscr{C}} \log \lambda S_{\lambda, \mathrm{sub}} d \lambda
$$

defines a classical $\psi$ do of order -1 , with symbol $s_{\mathrm{sub}}^{\log }\left(x^{\prime}, \xi^{\prime}\right)$ expanded in terms

$$
s_{\mathrm{sub},-j}^{\log }\left(x^{\prime}, \xi^{\prime}\right)=\frac{i}{2 \pi} \int_{\mathscr{C}} \log \lambda s_{-m-j}\left(x^{\prime}, \xi^{\prime}, \lambda\right) d \lambda, \quad j \geq 1 .
$$

When $v>1, S_{\text {sub }}^{\log }$ is the normal trace of $G_{\text {sub }}^{\log }$.

Proof. Since $G_{\lambda}$ and $G_{\lambda \text {,sub }}$ are of regularity $v \geq \frac{1}{2}, S_{\lambda}$ and $S_{\lambda, \text { sub }}$ are of regularity $v-\frac{1}{4} \geq \frac{1}{4}$, cf. [8, Section 3]. In particular, the symbols in $S_{\lambda, \text { sub }}$ are $O\left(\lambda^{-1-1 / 4 m}\right)$ on the rays in $V$ so that the integrals in (3.4) and (3.5) make sense.

As accounted for in the text before the theorem, there are estimates in the cases $v>1$ that allow interchange of the $\lambda$-integral with the $x_{n}$-integral involved in taking $\operatorname{tr}_{n}$.

For the operator in Example 2.3, we note that $S_{\lambda}=\operatorname{tr}_{n} G_{\lambda}$ is the $\psi$ do with symbol $-\left(2 \kappa_{1}\right)^{-2}=-\frac{1}{4}\left(\left\langle\xi^{\prime}\right\rangle^{2}-\lambda\right)^{-1}$, so its log-integral gives $-\frac{1}{4} \log \left(1-\Delta_{x^{\prime}}\right)$. This demonstrates that the "log-transform" of the principal part of $S_{\lambda}$ will not in general be a classical $\psi$ do.

Finally, we shall connect this with the study of the expansion coefficient $C_{0}\left(I,(P+G)_{T}\right)$ in the last section of [8]; we here write it simply as $C_{0}((P+$ $G)_{T}$ ) (or $\left.C_{0}(B)\right)$. It is known from [6, Sect. 3.3] that when $m>n$, the trace of the resolvent has an expansion in powers of $-\lambda$,

$$
\operatorname{Tr} R_{\lambda}=\sum_{0 \leq l \leq n} c_{l}(-\lambda)^{\frac{n-l}{m}-1}+O\left(\lambda^{-1-\frac{1}{4 m}}\right),
$$

and a similar proof shows that for general $m>0$, the expansion holds for a sufficiently high iterate:

$$
\operatorname{Tr} R_{\lambda}^{N}=\operatorname{Tr} \frac{\partial_{\lambda}^{N-1}}{(N-1) !} R_{\lambda}=\sum_{0 \leq l \leq n} c_{l}^{(N)}(-\lambda)^{\frac{n-l}{m}-N}+O\left(\lambda^{-N-\frac{1}{4 m}}\right) .
$$


Define the basic zeta value as the coefficient of $(-\lambda)^{-N}$ :

$$
C_{0}(B)=c_{n}^{(N)},
$$

it is independent of $N$. If $B$ is invertible, $C_{0}(B)$ equals the value of the zeta function $\zeta(B, s)-$ the meromorphic extension of $\operatorname{Tr}\left(B^{-s}\right)-$ at $s=0$. If $B$ has a nontrivial nullspace, the constants are connected by

$$
C_{0}(B)=\zeta(B, 0)+v_{0},
$$

where $v_{0}$ is the dimension of the generalized eigenspace of the zero eigenvalue.

There are similar expansions as in (3.7) of the traces of the $\psi$ do iterates $Q_{\lambda}^{N}$ on $\widetilde{X}$, truncated to $X$, that follow from integration over $X$ of the diagonal kernel expansions, as established in [6, Sect. 3.3] (with remarks); it is the s.g.o. contribution that presents the greater challenge in [6]. In view of the identifications in $\left[8\right.$, Sect. 1], the coefficient of $(-\lambda)^{-N}$ here equals $-\frac{1}{m} \operatorname{res}_{+}(\log P)$, where the plus-index indicates that the pointwise contribution to $-\frac{1}{m} \operatorname{res}(\log P)$ is integrated over $X$ only. It can also be regarded as $-\frac{1}{m} \operatorname{res}\left((\log P)_{+}\right)$, extending the notation of [4].

The constant $C_{0}(B)$ was analyzed in [8, Sect. 5] in relation to residue formulas, and we can now improve the result with further information.

THEOREM 3.2. One has that

$$
C_{0}(B)=-\frac{1}{m} \operatorname{res}_{+}(\log P)-\frac{1}{m} \operatorname{res}_{X^{\prime}}\left(S_{\text {sub }}^{\log }\right),
$$

where the terms are calculated as sums of contributions from local coordinate patches of the form

$$
\begin{aligned}
& \int_{\mathrm{R}_{+}^{n}} \int_{|\xi|=1} \operatorname{tr} l_{-n}(x, \xi) d S(\xi) d x, \quad \text { resp. } \\
& \int_{\mathrm{R}^{n-1}} \int_{\left|\xi^{\prime}\right|=1} \operatorname{tr} s_{\text {sub }, 1-n}^{\log }\left(x^{\prime}, \xi^{\prime}\right) d S\left(\xi^{\prime}\right) d x^{\prime} .
\end{aligned}
$$

The term $-\frac{1}{m} \operatorname{res}_{+}(\log P)$ has an invariant meaning as the coefficient of $(-\lambda)^{-N}$ in the expansion similar to (3.7) of $\operatorname{Tr}\left(\left((P-\lambda)^{-N}\right)_{+}\right)$, and hence the last term in the right-hand side of (3.10) likewise has an invariant meaning.

When the problem is differential, or when the problem is pseudodifferential with regularity $v>1$, then $\operatorname{res}_{X^{\prime}}\left(S_{\text {sub }}^{\log }\right)$ is, in local coordinates, the residue of the normal trace of $G_{\text {sub. }}^{\mathrm{log}}$. 
Proof. It was shown in $\left[8\right.$, Sect. 5] how $C_{0}(B)$ is found from integrals of the strictly homogeneous symbol terms of order $-m-n$ in $(P-\lambda)^{-1}$ resp. of order $-m-n+1$ in $G_{\lambda}$; the proof given for the case $m>n$ extends to general $m$ when the iterates are used, cf. [8, Remark 3.12]. It was shown moreover that these integrals by use of $[8$, Lemmas $1.2,1.3]$ could be turned into log-integrals as in (3.5). In those proofs, the log-integration is applied after the $\operatorname{tr}_{n}$-integration, so the boundary term is really $\operatorname{res}\left(S_{\text {sub }}^{\log }\right)$, as defined in Theorem 3.1.

When $v>1$, in particular when the problem is differential so that $v=\infty$, Theorem 3.1 shows that $S_{\text {sub }}^{\log }$ is the normal trace of $G_{\text {sub }}^{\log }$, so the assertion for the residues follows.

What we gain here in comparison with $[8$, Sect. 5] is a little more insight into how the boundary term stems from the s.g.o.-like part of $\log B$, plus the inclusion of all orders $m>0$. At any rate, since $C_{0}(B)$ is an invariant, we can propose it to be the residue of $-\frac{1}{m} \log B$ :

Definition 3.3. When $\left\{P_{+}+G-\lambda, T\right\}$ satisfies the hypotheses of parameter-ellipticity given above, the residue of $\log (P+G)_{T}$ is defined to be the constant

(3.12) $\operatorname{res}\left(\log (P+G)_{T}\right)=-m C_{0}\left((P+G)_{T}\right)=\operatorname{res}_{+}(\log P)+\operatorname{res}_{X^{\prime}}\left(S_{\text {sub }}^{\log }\right)$,

as calculated in Theorem 3.2.

This is consistent with the definition of [4]. We note that certain steps in an explicit calculation of this constant depend very much on localizations, e.g. in the steps of discarding the principal symbol and taking $\operatorname{tr}_{n}$. A number of similar or more general residue definitions are made in [9] for compositions of $\psi$ dbo's with components of $\log P_{T}$ (when $P_{T}$ is defined from an even-order differential problem). These residues do have a certain amount of traciality: $\operatorname{res}\left(\left[A, \log P_{T}\right]\right)=0$ holds for operators $A$ of order and class zero (cf. Theorem 6.5 there).

It should be noted that Definition 3.3 does not cover the case of first-order differential operators with spectral boundary conditions, since such boundary conditions are not normal. But for such boundary problems (Atiyah-PatodiSinger problems [1]) there exists a wealth of other treatments, adapted to the specific situation. The results there often depend on additional symmetry properties. (See e.g. [7] and its references.) 


\section{Sectorial projections}

Now we turn our attention to a certain spectral projection connected to the realization $(P+G)_{T}$; namely a projection whose range contains the closure of the direct sum of the generalized eigenspaces for the eigenvalues in a sector of the complex plane. Such projections have been studied earlier by Burak [3], Wodzicki [20], and Ponge [14]; the latter gives a detailed deduction of the basic properties in the case of classical $\psi$ do's on closed manifolds. We recall the properties below, supplying them with some additional information.

In order to apply the techniques to different types of operators, we first consider an abstract situation where $A$ denotes an unbounded, densely defined, closed operator in a Hilbert space $H$. It is assumed to have the following properties:

$A$ has a resolvent set containing two sectors $V_{\theta}$ and $V_{\varphi}$ around $e^{i \theta} \mathrm{R}_{+}$and $e^{i \varphi} \mathbf{R}_{+}$, respectively, for some $\theta<\varphi<\theta+2 \pi$, the resolvent $(A-\lambda)^{-1}$ is compact, and $\left\|(A-\lambda)^{-1}\right\|$ is $O\left(\lambda^{-1}\right)$ for $\lambda$ going to infinity on each ray of these sectors. (We refer to Kato [12] for general background theory.)

For $x \in D(A)$ and $\lambda$ on a ray in either sector, we have

$$
\left\|\lambda^{-1} A(A-\lambda)^{-1} x\right\| \leq\left\|\lambda^{-1}(A-\lambda)^{-1}\right\| \cdot\|A x\|=O\left(\lambda^{-2}\right),
$$

so that $\lambda^{-1} A(A-\lambda)^{-1} x$ is integrable for $|\lambda| \rightarrow \infty$.

Then define the operator $\Pi_{\theta, \varphi}(A)$, the sectorial projection, with domain $D(A)$ to begin with, by

$$
\Pi_{\theta, \varphi}(A) x=\frac{i}{2 \pi} \int_{\Gamma_{\theta, \varphi}} \lambda^{-1} A(A-\lambda)^{-1} x d \lambda, \quad x \in D(A),
$$

where the integration goes along the sectorial contour

$$
\Gamma_{\theta, \varphi}=\left\{r e^{i \varphi} \mid \infty>r>r_{0}\right\} \cup\left\{r_{0} e^{i \omega} \mid \varphi \geq \omega \geq \theta\right\} \cup\left\{r e^{i \theta} \mid r_{0}<r<\infty\right\},
$$

with $r_{0}$ taken so small that 0 is the only possible eigenvalue in $\left\{|\lambda|<r_{0}\right\}$. If the operator is bounded in $H$-norm, we extend it to $H$. This operator is a spectral projection in the following sense:

For each $\lambda \in \sigma(A)$, denote the generalized eigenspace by $E_{\lambda}$,

$$
E_{\lambda}=\bigcup_{k \in \mathrm{N}} \operatorname{ker}(A-\lambda)^{k}
$$

(it equals $\operatorname{ker}(A-\lambda)^{k_{0}}$ for a sufficiently large $\left.k_{0}\right)$. For $\alpha<\beta$, set

$$
\Lambda_{\alpha, \beta}=\left\{r e^{i \omega} \mid r>0, \alpha<\omega<\beta,\right\}, \quad E_{\alpha, \beta}=\dot{+}_{\lambda \in \sigma(A) \cap \Lambda_{\alpha, \beta}} E_{\lambda} .
$$


Proposition 4.1. $\Pi_{\theta, \varphi}(A)^{2}=\Pi_{\theta, \varphi}(A)$, i.e. $\Pi_{\theta, \varphi}(A)$ is a (possibly unbounded) projection in $H$. Its range contains $E_{\theta, \varphi}$ and its kernel contains $E_{0}+E_{\varphi, \theta+2 \pi}$.

(a) If $A$ has a complete system of root vectors, i.e. $\dot{+}_{\lambda \in \sigma(A)} E_{\lambda}$ is dense in $H$, then $\Pi_{\theta, \varphi}(A)$ is the bounded projection onto $\overline{E_{\theta, \varphi}}$ along $E_{0}+\overline{E_{\varphi, \theta+2 \pi}}$.

(b) If A is normal, i.e. $A^{*} A=A A^{*}$, then $\Pi_{\theta, \varphi}(A)$ is the bounded orthogonal projection onto $\oplus_{\lambda \in \sigma(A) \cap \Lambda_{\theta, \varphi}} \operatorname{ker}(A-\lambda)$ along $\oplus_{\lambda \in \sigma(A) \backslash \Lambda_{\theta, \varphi}} \operatorname{ker}(A-\lambda)$.

PRoof. Except for a few elementary considerations regarding the domain and closedness, the proofs of [14, Propositions 3.2, A.4, and A.5] carry over almost word for word to the present setting (it should be noted that some contours in [14] have the opposite orientation).

In (a) and (b), the boundedness of $\Pi_{\theta, \varphi}(A)$ follows from the fact that the kernel and range are closed.

In certain important cases, $\Pi_{\theta, \varphi}(A)$ can be seen to be bounded regardless of whether the hypotheses of (a) or (b) can be verified; as shown in [14, Proposition 3.1] this holds when $A$ is a $\psi$ do of order $m>0$ on a closed manifold. We shall see below in Theorem 4.6 that it also holds for the realization of a differential elliptic boundary value problem.

As shown below, the sectorial projection has a direct connection with the choice of spectral cut in our definition of the logarithm of an operator. Using arguments as in Section 2, we can define the logarithm of $A$ with a branch cut at the angle $\theta$ as

$$
\log _{\theta} A=\lim _{s \searrow 0} \frac{i}{2 \pi} \int_{\mathscr{C}_{\theta}} \lambda_{\theta}^{-s} \log _{\theta} \lambda(A-\lambda)^{-1} d \lambda
$$

where the subscript $\theta$ indicates that $\lambda^{-s} \log \lambda$ is chosen to have a branch cut along $e^{i \theta} \mathbf{R}_{+}$, and the contour is the Laurent loop

$$
\begin{aligned}
& \mathscr{C}_{\theta}=\left\{r e^{i \theta} \mid \infty>r>r_{0}\right\} \\
& \cup\left\{r_{0} e^{i \omega} \mid \theta \geq \omega \geq \theta-2 \pi\right\} \cup\left\{r e^{i(\theta-2 \pi)} \mid r_{0}<r<\infty\right\} .
\end{aligned}
$$

The following proposition eliminates the limiting procedure of (4.4) and gives a useful alternative description of $\Pi_{\theta, \varphi}(A)$. A proof can be found in the Appendix.

Proposition 4.2. For $x \in D(A)$ we have the identities

$$
\begin{aligned}
\log _{\theta} A x & =\frac{i}{2 \pi} \int_{\mathscr{C}_{\theta}} \lambda^{-1} \log _{\theta} \lambda A(A-\lambda)^{-1} x d \lambda \quad \text { and } \\
\Pi_{\theta, \varphi}(A) x & =\frac{i}{2 \pi} \int_{\Gamma_{\theta, \varphi}}(A-\lambda)^{-1} x d \lambda+\frac{\varphi-\theta}{2 \pi} x,
\end{aligned}
$$


where the integral in the right-hand side of (4.7) is an improper integral.

Next, we include a lemma which will be useful for our considerations regarding expressions involving different branches of the logarithm. Again, a proof is available in the Appendix.

Lemma 4.3. Let $f(\lambda)$ be a continuous (possibly vector-valued) function on the "punctuated double keyhole region"

$$
V_{r_{0}, \delta}=\left\{\lambda \in \mathrm{C}|| \lambda \mid<2 r_{0} \text { or }|\arg \lambda-\theta|<\delta \text { or }|\arg \lambda-\varphi|<\delta\right\} \backslash\{0\},
$$

such that $f(\lambda)$ is $O\left(\lambda^{-1-\varepsilon}\right)$ for $|\lambda| \rightarrow \infty$ in $V_{r_{0}, \delta}$. Then

$$
\int_{\mathscr{C}_{\theta}} \log _{\theta} \lambda f(\lambda) d \lambda-\int_{\mathscr{C}_{\varphi}} \log _{\varphi} \lambda f(\lambda) d \lambda=-2 \pi i \int_{\Gamma_{\theta, \varphi}} f(\lambda) d \lambda .
$$

We can use this lemma to describe the relation between $\Pi_{\theta, \varphi}(A)$ and $\log$ arithms of $A$ as follows:

Proposition 4.4. For $x \in D(A)$,

$$
\log _{\theta} A x-\log _{\varphi} A x=\int_{\Gamma_{\theta, \varphi}} \lambda^{-1} A(A-\lambda)^{-1} x d \lambda=-2 \pi i \Pi_{\theta, \varphi}(A) x
$$

When $\Pi_{\theta, \varphi}(A)$ is bounded, so is $\log _{\theta} A-\log _{\varphi} A$, and

$$
\Pi_{\theta, \varphi}(A)=\frac{i}{2 \pi}\left(\log _{\theta} A-\log _{\varphi} A\right) .
$$

Proof. For $x \in D(A)$, the expression $f(\lambda)=\lambda^{-1} A(A-\lambda)^{-1} x$ is holomorphic in $V_{r_{0}, \delta}$ for some $r_{0}, \delta>0$, and $f(\lambda)$ is $O\left(\lambda^{-2}\right)$ for $|\lambda| \rightarrow \infty$ in $V_{r_{0}, \delta}$ by (4.1).

Hence we can apply Lemma 4.3, and insertion of the expression for $f(\lambda)$ into (4.9) gives

$$
\begin{aligned}
\int_{\mathscr{C}_{\theta}} \log _{\theta} \lambda \lambda^{-1} A(A-\lambda)^{-1} x d \lambda & -\int_{\mathscr{C}_{\varphi}} \log _{\varphi} \lambda \lambda^{-1} A(A-\lambda)^{-1} x d \lambda \\
& =-2 \pi i \int_{\Gamma_{\theta, \varphi}} \lambda^{-1} A(A-\lambda)^{-1} x d \lambda .
\end{aligned}
$$

Then (4.10) follows from (4.2) and (4.6).

If $\Pi_{\theta, \varphi}(A)$ is bounded, (4.10) extends to all $x \in H$ since $D(A)$ is dense in $H$, and (4.11) follows. 
With the results above at hand we return to the realization $(P+G)_{T}$. Modifying the assumption of Section 2 a little, we now assume $\left\{P_{+}+G-\lambda, T\right\}$ to satisfy the conditions of parameter-ellipticity in [6, Def. 3.3.1] for $\lambda$ on the rays of two sectors around $e^{i \theta} \mathbf{R}_{+}$and $e^{i \varphi} \mathbf{R}_{+}$, respectively. Then the realization $B=(P+G)_{T}$ satisfies the requirements for $A$ above (4.1), and we can define the sectorial projection accordingly:

$$
\Pi_{\theta, \varphi}(B)=\frac{i}{2 \pi} \int_{\Gamma_{\theta, \varphi}} \lambda^{-1} B R_{\lambda} d \lambda .
$$

Here, and below, the integrals are understood to be in the strong sense, to simplify notation. Like in the case of the logarithm, we decompose it into the contributions from the pseudodifferential and singular Green parts.

For the $\psi$ do $P$ on the closed manifold $\widetilde{X}$, we can use Proposition 4.2 to see that

$$
\frac{i}{2 \pi} \int_{\Gamma_{\theta, \varphi}} Q_{\lambda} u d \lambda+\frac{\varphi-\theta}{2 \pi} u=\Pi_{\theta, \varphi}(P) u, \quad u \in D(P)
$$

it is known from [20], [14], that $\Pi_{\theta, \varphi}(P)$ is a $\psi$ do of order $\leq 0$ on $\tilde{X}$.

Using Proposition 4.2, (2.4), and the fact that $r^{+} e^{+}=I$, we can rewrite (4.13) as

$$
\begin{aligned}
\Pi_{\theta, \varphi}(B) & =\frac{i}{2 \pi} \int_{\Gamma_{\theta, \varphi}} R_{\lambda} d \lambda+\frac{\varphi-\theta}{2 \pi} \\
& =\frac{i}{2 \pi} \int_{\Gamma_{\theta, \varphi}}\left[Q_{\lambda,+}+G_{\lambda}\right] d \lambda+\frac{\varphi-\theta}{2 \pi} \\
& =r^{+}\left(\frac{i}{2 \pi} \int_{\Gamma_{\theta, \varphi}} Q_{\lambda} d \lambda+\frac{\varphi-\theta}{2 \pi}\right) e^{+}+\frac{i}{2 \pi} \int_{\Gamma_{\theta, \varphi}} G_{\lambda} d \lambda \\
& =\Pi_{\theta, \varphi}(P)_{+}+\frac{i}{2 \pi} \int_{\Gamma_{\theta, \varphi}} G_{\lambda} d \lambda ;
\end{aligned}
$$

in the last line we moreover used (4.14). Now an application of Proposition 4.4 to $P$ and $B$ gives:

$$
\begin{aligned}
\Pi_{\theta, \varphi}(P)_{+} & =\frac{i}{2 \pi}\left(\left(\log _{\theta} P\right)_{+}-\left(\log _{\varphi} P\right)_{+}\right), \\
\Pi_{\theta, \varphi}(B) & =\frac{i}{2 \pi}\left(\log _{\theta} B-\log _{\varphi} B\right) .
\end{aligned}
$$


Using the contour $\mathscr{C}_{\theta}$ from (4.5) we can define an operator as in (2.13),

$$
G^{\log _{\theta}}=\frac{i}{2 \pi} \int_{\mathscr{C}_{\theta}} \log _{\theta} \lambda G_{\lambda} d \lambda,
$$

and similarly define $G^{\log _{\varphi}}$ where $\theta$ is replaced by $\varphi$. By rotation it is obvious that $G^{\log _{\theta}}$ and $G^{\log _{\varphi}}$ have properties similar to those of $G^{\log }$ described in Section 2. Now (4.16) and (2.15) show that if we define $G_{\theta, \varphi}$ by

$$
G_{\theta, \varphi}=\frac{i}{2 \pi} \int_{\Gamma_{\theta, \varphi}} G_{\lambda} d \lambda
$$

then

$$
G_{\theta, \varphi}=\frac{i}{2 \pi}\left(G^{\log _{\theta}}-G^{\log _{\varphi}}\right) .
$$

In view of (4.15), we have then obtained:

THeOREM 4.5. The sectorial projection for $B=(P+G)_{T}$ satisfies

$$
\Pi_{\theta, \varphi}(B)=\Pi_{\theta, \varphi}(P)_{+}+G_{\theta, \varphi},
$$

where each term on the right hand side is known: $\Pi_{\theta, \varphi}(P)_{+}$is the truncation of a $\psi$ do on $\widetilde{X}$ of order at most zero, in particular it is bounded on $L_{2}(X, E)$; $G_{\theta, \varphi}$ is a difference (4.19) of two terms of the log-type described in Section 2 and hence is a generalized singular Green operator, bounded from $L_{2}(X, E)$ to $H^{-\varepsilon}(X, E)$.

Like $G^{\log }, G_{\theta, \varphi}$ acts as in (2.21). It has a symbol-kernel $\tilde{g}_{\theta, \varphi} \sim \sum_{j \in \mathbf{N}} \tilde{g}_{\theta, \varphi,-j}$, with terms given by

$$
\begin{aligned}
\tilde{g}_{\theta, \varphi,-j} & =\frac{i}{2 \pi}\left(\tilde{g}_{-j}^{\log _{\theta}}-\tilde{g}_{-j}^{\log _{\varphi}}\right) \\
& =\frac{-1}{4 \pi^{2}}\left(\int_{\mathscr{C}_{\theta}} \log _{\theta} \lambda \tilde{g}_{-m-j} d \lambda-\int_{\mathscr{C}_{\varphi}} \log _{\varphi} \lambda \tilde{g}_{-m-j} d \lambda\right) .
\end{aligned}
$$

By Lemma 4.3 this is simplified to

$$
\tilde{g}_{\theta, \varphi,-j}\left(x^{\prime}, x_{n}, y_{n}, \xi^{\prime}\right)=\frac{i}{2 \pi} \int_{\Gamma_{\theta, \varphi}} \tilde{g}_{-m-j}\left(x^{\prime}, x_{n}, y_{n}, \xi^{\prime}, \lambda\right) d \lambda .
$$

In view of (4.19) and (4.21), the results on $G^{\log }$ resp. $\tilde{g}^{\log }$ in Section 2 carry over immediately to $G_{\theta, \varphi}$ resp. $\tilde{g}_{\theta, \varphi}$. We shall not reproduce all the statements 
explicitly, but will just present the following important result obtained from Theorem 2.4.

Theorem 4.6. Assume that $P$ is a differential operator, $G=0$, and the trace operators $T_{0}, \ldots, T_{m-1}$ are differential operators; hereby $B=P_{T}$.

Then $G_{\theta, \varphi}$ is, in local coordinates near $X^{\prime}$, a generalized singular Green operator

$$
G_{\theta, \varphi}=\operatorname{OPG}\left(\tilde{g}_{\theta, \varphi}\right)
$$

with $\tilde{g}_{\theta, \varphi} \sim \sum_{j \in \mathrm{N}} \tilde{g}_{\theta, \varphi,-j}$; the $j$ 'th term is quasihomogeneous as in (2.22) and satisfies estimates as in (2.23).

$G_{\theta, \varphi}$ and $\Pi_{\theta, \varphi}\left(P_{T}\right)$ are bounded operators in $L_{p}(X, E)$ for $1<p<\infty$. In particular, $\Pi_{\theta, \varphi}\left(P_{T}\right)$ is a bounded projection in $L_{2}(X, E)$.

Proof. The claims regarding $\tilde{g}_{\theta, \varphi}$ follow immediately from Theorem 2.4 and (4.21).

The boundedness properties of $G_{\theta, \varphi}$ are obvious from Theorem 2.4 and (4.19). Since $\Pi_{\theta, \varphi}(P)_{+}$is the truncation of a $\psi$ do of order at most zero, this is also bounded in $L_{p}(X, E)$; then in view of (4.20) so is $\Pi_{\theta, \varphi}\left(P_{T}\right)$.

An interesting question is whether one can give criteria on $P, G$, and $T$ assuring that the operator $\Pi_{\theta, \varphi}\left((P+G)_{T}\right)$ belongs to the Boutet de Monvel calculus.

Concerning the $\psi$ do part $\Pi_{\theta, \varphi}(P)$, with symbol $\pi_{\theta, \varphi}(x, \xi)$ in local coordinates, we have easily by use of Lemma 2.1 :

Lemma 4.7. When $m$ is even, $\pi_{\theta, \varphi}(x, \xi)$ satisfies the transmission condition.

Hence $\Pi_{\theta, \varphi}(P)_{+}$is in the Boutet de Monvel calculus for even $m$.

Proof. We have that in view of (2.10) that

$$
\operatorname{symb}\left(\log _{\theta} P\right)=m \log [\xi]+l_{\theta}(x, \xi), \quad l_{\theta}(x, \xi) \sim \sum_{j \in \mathrm{N}} l_{\theta,-j}(x, \xi),
$$

where $m \log [\xi]+l_{\theta, 0}(x, \xi)=\log _{\theta}\left(p_{m}(x, \xi)\right)$, with similar formulas for $\log _{\varphi} P$, so the symbols of $\log _{\theta} P$ and $\log _{\varphi} P$ have the same $\log$-term $m \log [\xi]$. Then it is seen from the first line in (4.16) that

$$
\pi_{\theta, \varphi}(x, \xi)=\frac{i}{2 \pi}\left(l_{\theta}(x, \xi)-l_{\varphi}(x, \xi)\right),
$$

which satisfies the transmission condition when $m$ is even in view of Lemma 2.1. 
This could also be based more directly on the fact, worked out in detail in [14], that $\pi_{\theta, \varphi}(x, \xi) \sim \sum_{j \in \mathbb{N}} \pi_{\theta, \varphi,-j}(x, \xi)$, where the terms are given by

$$
\pi_{\theta, \varphi,-j}(x, \xi)=\frac{i}{2 \pi} \int_{\mathscr{C}_{\theta, \varphi}(x, \xi)} q_{-m-j}(x, \xi, \lambda) d \lambda ;
$$

here $\mathscr{C}_{\theta, \varphi}(x, \xi)$ is a closed curve in the sector $\Lambda_{\theta, \varphi}$ going in the positive direction around the part of the spectrum of $p_{m}(x, \xi)$ lying in that sector.

When $m$ is odd, one cannot expect $\Pi_{\theta, \varphi}(P)$ to satisfy the transmission condition. For example, for a first-order selfadjoint invertible elliptic differential operator $A$ on $\widetilde{X}$ (e.g., a Dirac operator), $\Pi_{-\frac{\pi}{2}, \frac{\pi}{2}}(A)$ equals $\Pi_{>}(A)$, the positive eigenprojection $\frac{1}{2}\left(I+A\left|A^{2}\right|^{-1 / 2}\right)$, where $A\left|A^{2}\right|^{-1 / 2}$ does not satisfy the transmission condition (its even-order symbol terms are odd in $\xi$ ).

Next, let us consider the s.g.o. part $G_{\theta, \varphi}$. Example 4.8 below shows a differential operator realization where $G_{\theta, \varphi}$ is not a standard singular Green operator, already in a constant-coefficient principal symbol case. Example 4.9 on the other hand defines a general class of differential operator realizations where $G_{\theta, \varphi}$ is a standard s.g.o., and $\Pi_{\theta, \varphi}(B)$ belongs to the standard calculus. Here one finds however, that lower order perturbations can ruin the standard s.g.o.-properties.

Example 4.8. Consider the differential operators $A$ and $P$ on $\mathrm{R}_{+}^{4}$ given by

$$
A=\left(\begin{array}{rr}
i & 0 \\
0 & -i
\end{array}\right) D_{1}+\left(\begin{array}{rr}
0 & 1 \\
-1 & 0
\end{array}\right) D_{2}+\left(\begin{array}{ll}
0 & i \\
i & 0
\end{array}\right) D_{3}+\left(\begin{array}{ll}
1 & 0 \\
0 & 1
\end{array}\right) D_{4}
$$

and

$$
P=\left(\begin{array}{cc}
0 & -A^{*} \\
A & 0
\end{array}\right),
$$

where $A^{*}$ denotes the formal adjoint of $A$. ( $A$ and $P$ are Dirac-type operators, with $A^{*} A=-\Delta I_{2},(i P)^{2}=-\Delta I_{4}$.)

Regarding this as a localization of a manifold situation, we seek the projection onto the (generalized) eigenspaces for the eigenvalues $\lambda$ in the upper halfplane $\mathrm{C}_{+}$for a certain realisation $P_{T}$ of $P$, where the boundary condition is $B \gamma_{0} u=0$, with

$$
B=\left(\begin{array}{llll}
1 & 0 & 1 & 0 \\
0 & 1 & 0 & 1
\end{array}\right)
$$

i.e., $\gamma_{0} u_{1}+\gamma_{0} u_{3}=\gamma_{0} u_{2}+\gamma_{0} u_{4}=0, u_{i}$ being the $i$ 'th component of $u$. 
Thus, in this localized situation we shall construct $\Pi_{\theta, \varphi}\left(P_{T}\right)$ with $\theta=0$ and $\varphi=\pi$. In this case the contour $\Gamma_{\theta, \varphi}$ is a contour from $-\infty$ to $\infty$ passing above the origin.

$P$ has symbol

$$
\begin{aligned}
p(\xi) & =\left(\begin{array}{cccc}
0 & -{ }^{t} \overline{a(\xi)} \\
a(\xi) & 0
\end{array}\right) \\
& =\left(\begin{array}{cccc}
0 & 0 & i \xi_{1}-\xi_{4} & \xi_{2}+i \xi_{3} \\
0 & 0 & -\xi_{2}+i \xi_{3} & -i \xi_{1}-\xi_{4} \\
i \xi_{1}+\xi_{4} & \xi_{2}+i \xi_{3} & 0 & 0 \\
-\xi_{2}+i \xi_{3} & -i \xi_{1}+\xi_{4} & 0 & 0
\end{array}\right),
\end{aligned}
$$

the eigenvalues of which are $\pm i|\xi|$. Hence $P-\lambda$ is parameter-elliptic for $\lambda$ on all rays in $\mathrm{C} \backslash i \mathrm{R}$, with parametrix-symbol

$$
\begin{aligned}
q(\xi, \lambda) & =(p(\xi)-\lambda)^{-1} \\
& =\frac{1}{|\xi|^{2}+\lambda^{2}}\left(\begin{array}{cccc}
-\lambda & 0 & -i \xi_{1}+\xi_{4} & -\xi_{2}-i \xi_{3} \\
0 & -\lambda & \xi_{2}-i \xi_{3} & i \xi_{1}+\xi_{4} \\
-i \xi_{1}-\xi_{4} & -\xi_{2}-i \xi_{3} & -\lambda & 0 \\
\xi_{2}-i \xi_{3} & i \xi_{1}-\xi_{4} & 0 & -\lambda
\end{array}\right) .
\end{aligned}
$$

We first find the $\psi$ do part of $\Pi_{0, \pi}\left(P_{T}\right)$ : According to (4.26) the symbol $\pi(\xi)$ of $\Pi_{0, \pi}(P)$ is obtained by integrating $q(\xi, \lambda)$ along a small closed curve, $\mathscr{C}_{\xi}$, enclosing the pole $i|\xi|$ in $\mathrm{C}_{+}$:

$$
\begin{aligned}
\pi(\xi) & =\frac{i}{2 \pi} \int_{\mathscr{C}_{\xi}} q(\xi, \lambda) d \lambda=-\underset{\lambda=i|\xi|}{\operatorname{Res}}(q(\xi, \lambda)) \\
& =\frac{1}{2|\xi|}\left(\begin{array}{cccc}
|\xi| & 0 & \xi_{1}+i \xi_{4} & -i \xi_{2}+\xi_{3} \\
0 & |\xi| & i \xi_{2}+\xi_{3} & -\xi_{1}+i \xi_{4} \\
\xi_{1}-i \xi_{4} & -i \xi_{2}+\xi_{3} & |\xi| & 0 \\
i \xi_{2}+\xi_{3} & -\xi_{1}-i \xi_{4} & 0 & |\xi|
\end{array}\right) .
\end{aligned}
$$

The singular Green part $G_{\lambda}$ of the resolvent $R_{\lambda}=\left(P_{T}-\lambda\right)^{-1}$ has symbolkernel

$\tilde{g}\left(x_{n}, y_{n}, \xi^{\prime}, \lambda\right)$ 


$$
=\frac{1}{2 \sigma}\left(\begin{array}{cccc}
-i \xi_{1}+i \sigma & -\xi_{2}-i \xi_{3} & -\lambda & 0 \\
\xi_{2}-i \xi_{3} & i \xi_{1}+i \sigma & 0 & -\lambda \\
-\lambda & 0 & -i \xi_{1}-i \sigma & -\xi_{2}-i \xi_{3} \\
0 & -\lambda & \xi_{2}-i \xi_{3} & i \xi_{1}-i \sigma
\end{array}\right) e^{-\sigma\left(x_{n}+y_{n}\right)},
$$

where $\sigma=\sqrt{\left|\xi^{\prime}\right|^{2}+\lambda^{2}}$. Note that $\sigma$ is holomorphic (and $\operatorname{Re} \sigma>0$ ) for $\lambda \in \mathrm{C} \backslash \pm i\left(\left|\xi^{\prime}\right|, \infty\right)$; in particular $\left\{P-\lambda, B \gamma_{0}\right\}$ is parameter-elliptic for $\lambda$ on any ray in $\mathrm{C} \backslash i \mathrm{R}$.

The integration contour $\Gamma_{0, \pi}$ is homotopic in $\left\{r e^{i \omega} \mid \omega \neq \pm \frac{\pi}{2}\right.$ or $\left.r<\left|\xi^{\prime}\right|\right\}$ to the real line; thus, due to the exponential falloff of $e^{-\left(\left|\xi^{\prime}\right|^{2}+\lambda^{2}\right)^{\frac{1}{2}}\left(x_{n}+y_{n}\right)}$ we get

$$
\begin{aligned}
\tilde{g}_{\theta, \varphi}\left(x_{n}, y_{n}, \xi^{\prime}\right) & =\frac{i}{2 \pi} \int_{\Gamma_{\theta, \varphi}} \tilde{g}\left(x_{n}, y_{n}, \xi^{\prime}, \lambda\right) d \lambda \\
& =\frac{i}{2 \pi} \int_{-\infty}^{\infty} \tilde{g}\left(x_{n}, y_{n}, \xi^{\prime}, t\right) d t .
\end{aligned}
$$

We can now verify that $\tilde{g}_{\theta, \varphi}$ is not a singular Green symbol-kernel: The 12matrix entry of $\tilde{g}_{\theta, \varphi}$ becomes

$$
\frac{-i \xi_{2}+\xi_{3}}{4 \pi} \int_{-\infty}^{\infty}\left(\left|\xi^{\prime}\right|^{2}+t^{2}\right)^{-\frac{1}{2}} e^{-\left(\left|\xi^{\prime}\right|^{2}+t^{2}\right)^{\frac{1}{2}}\left(x_{n}+y_{n}\right)} d t,
$$

which, for fixed $\xi^{\prime}$, is unbounded as $x_{n}+y_{n}$ goes to zero; hence, $\tilde{g}_{\theta, \varphi}$ is not in $\mathscr{S}_{++}$.

To see this note that, for fixed $a>0$,

$$
\begin{aligned}
f(r) & =\frac{1}{2} \int_{-\infty}^{\infty}\left(a^{2}+t^{2}\right)^{-\frac{1}{2}} e^{-r\left(a^{2}+t^{2}\right)^{\frac{1}{2}}} d t=\int_{0}^{\infty}\left(a^{2}+t^{2}\right)^{-\frac{1}{2}} e^{-r\left(a^{2}+t^{2}\right)^{\frac{1}{2}}} d t \\
& \geq \int_{0}^{\infty} \frac{e^{-(a+t) r}}{a+t} d t=\int_{a r}^{\infty} \frac{e^{-u}}{u} d u
\end{aligned}
$$

which diverges to $+\infty$ as $r \rightarrow 0^{+}$.

EXAmple 4.9. Let $X_{0}^{\prime}$ be a closed $(n-1)$-dimensional manifold provided with an elliptic second-order differential operator $S$ which is selfadjoint positive in $L_{2}\left(X_{0}^{\prime}\right)$. Let $X=X_{0}^{\prime} \times[0, a]$ with points $x=\left(x^{\prime}, x_{n}\right), x^{\prime} \in X_{0}^{\prime}$ and $x_{n} \in[0, a]$, and let $B$ be the Dirichlet realization of $D_{x_{n}}^{2}+S$ on $X$; it is selfadjoint positive in $L_{2}(X)$, with $D(B)=H^{2}(X) \cap H_{0}^{1}(X)$. Let $A$ be the Dirichlet realization of

$$
P=\left(\begin{array}{cc}
D_{x_{n}}^{2}+S & S \\
S & -D_{x_{n}}^{2}-S
\end{array}\right) .
$$


on $X$, then in fact,

$$
A=\left(\begin{array}{rr}
B & S \\
S & -B
\end{array}\right)
$$

with domain $D(B) \times D(B)$. The resolvent is

$$
(A-\lambda)^{-1}=\left(\begin{array}{cc}
-B-\lambda & -S \\
-S & B-\lambda
\end{array}\right)\left(\lambda^{2}-B^{2}-S^{2}\right)^{-1},
$$

where we used that $S$ and $B$ commute. Define $B_{1}=\left(B^{2}+S^{2}\right)^{\frac{1}{2}}$. Here $B^{2}+S^{2}$ is the realization of the fourth-order elliptic differential operator $\left(D_{x_{n}}^{2}+S\right)^{2}+S^{2}$ determined by the boundary condition $\gamma_{0} u=0, \gamma_{0} B u=0$. This is one of the particular cases where the square root of the interior operator does satisfy the transmission condition, cf. [6, (4.4.9)]. Moreover, the square root of the realization $B^{2}+S^{2}$ represents a boundary condition consisting of exactly the part of the boundary condition for $B^{2}+S^{2}$ that makes sense on $H^{2}(X)$, cf. [6, Cor. 4.4.3] (based on a result of Grisvard); so in fact $B_{1}$ is the realization of $\left(\left(D_{x_{n}}^{2}+S\right)^{2}+S^{2}\right)^{\frac{1}{2}}$ determined by the Dirichlet condition $\gamma_{0} u=0$. This belongs to the standard calculus and enters nicely in the theory of [6], cf. Section 1.7 there. Note that $D\left(B_{1}\right)=D(B)$.

We can then calculate

$$
\begin{aligned}
\left(\lambda^{2}-\right. & \left.\left(B^{2}+S^{2}\right)\right)^{-1} \\
& =\left(\lambda^{2}-B_{1}^{2}\right)^{-1}=\left(B_{1}-\lambda\right)^{-1}\left(-B_{1}-\lambda\right)^{-1} \\
& =\left(B_{1}-\lambda\right)^{-1}\left(2 B_{1}\right)^{-1}\left(B_{1}+\lambda+B_{1}-\lambda\right)\left(-B_{1}-\lambda\right)^{-1}, \\
& =-\frac{1}{2} B_{1}^{-1}\left(\left(B_{1}-\lambda\right)^{-1}-\left(-B_{1}-\lambda\right)^{-1}\right)
\end{aligned}
$$

which leads to the formula:

$$
\begin{aligned}
(A-\lambda)^{-1} & \\
= & \left(\begin{array}{cc}
-B+B_{1}-B_{1}-\lambda & -S \\
-S & B-B_{1}+B_{1}-\lambda
\end{array}\right)\left(B_{1}-\lambda\right)^{-1}\left(-B_{1}-\lambda\right)^{-1} \\
= & \left(\begin{array}{cc}
\left(B_{1}-\lambda\right)^{-1} & 0 \\
0 & \left(-B_{1}-\lambda\right)^{-1}
\end{array}\right) \\
& -\left(\begin{array}{cc}
B_{1}-B & -S \\
-S & B-B_{1}
\end{array}\right) \frac{1}{2} B_{1}^{-1}\left(\left(B_{1}-\lambda\right)^{-1}-\left(-B_{1}-\lambda\right)^{-1}\right),
\end{aligned}
$$

valid for $\lambda$ outside the spectra of $B_{1}$ and $-B_{1}$. To determine the spectral projection $\Pi_{\theta, \varphi}(A)$ with $\theta=-\frac{\pi}{2}, \varphi=\frac{\pi}{2}$, we use the abstract machinery. It is 
seen from either of the formulas (4.2) or (4.7) that

$$
\begin{aligned}
& \Pi_{-\frac{\pi}{2}, \frac{\pi}{2}}(A)=\left(\begin{array}{cc}
\Pi_{-\frac{\pi}{2}, \frac{\pi}{2}}\left(B_{1}\right) & 0 \\
0 & \Pi_{-\frac{\pi}{2}, \frac{\pi}{2}}\left(-B_{1}\right)
\end{array}\right) \\
& \quad-\left(\begin{array}{cc}
B_{1}-B & -S \\
-S & B-B_{1}
\end{array}\right) \frac{1}{2} B_{1}^{-1}\left(\Pi_{-\frac{\pi}{2}, \frac{\pi}{2}}\left(B_{1}\right)-\Pi_{-\frac{\pi}{2}, \frac{\pi}{2}}\left(-B_{1}\right)\right) .
\end{aligned}
$$

Here

$$
\Pi_{-\frac{\pi}{2}, \frac{\pi}{2}}\left(B_{1}\right)=I, \quad \Pi_{-\frac{\pi}{2}, \frac{\pi}{2}}\left(-B_{1}\right)=0,
$$

in view of Proposition 4.1 and the fact that $B_{1}$ is selfadjoint positive. It follows that

$$
\Pi_{-\frac{\pi}{2}, \frac{\pi}{2}}(A)=\left(\begin{array}{cc}
\frac{1}{2}+\frac{1}{2} B B_{1}^{-1} & \frac{1}{2} S B_{1}^{-1} \\
\frac{1}{2} S B_{1}^{-1} & \frac{1}{2}-\frac{1}{2} B B_{1}^{-1}
\end{array}\right) .
$$

The operator is in the Boutet de Monvel calculus. Note that the sum of the diagonal terms is $I$, so the residue of the operator is zero.

Inherent in this example are some symbol calculations where the poles of the resolvent symbol appear isolated in such a way that integrals over $\Gamma_{\theta, \varphi}$ can be turned into integrals over closed curves, reducing to simple residue calculations. Perturbations can easily introduce more complicated calculations where integrals as in (4.32) appear, leading to non-standard s.g.o.-symbols (we shall not reproduce examples here).

In view of Definition 3.3 and the formulas (4.16), the sectorial projection $\Pi_{\theta, \varphi}(B)$ has a well-defined residue. In the differential operator case where the order $m$ is even, one can moreover define residues of the compositions of $\Pi_{\theta, \varphi}(B)$ with operators $A$ in the Boutet de Monvel calculus; this is taken up in [9]. It is found there that if in addition, $A$ is of order and class 0 , the residue vanishes on the commutator of $\Pi_{\theta, \varphi}(B)$ and $A$.

It is still an open question whether the residue is zero on sectorial projections for boundary value problems, as it is in the closed manifold case; we expect to return to this question in a forthcoming work.

\section{Appendix A. Proofs of auxiliary results in functional analysis}

Proof of Proposition 4.2. First we prove (4.6): Let, for $N \in \mathrm{N}$,

$$
\text { (A.1) } \begin{aligned}
& \mathscr{C}_{\theta}^{N}=\left\{r e^{i \theta} \mid N \geq r \geq r_{0}\right\} \\
& \cup\left\{r_{0} e^{i \omega} \mid \theta \geq \omega \geq \theta-2 \pi\right\} \cup\left\{r e^{i(\theta-2 \pi)} \mid r_{0} \leq r \leq N\right\} .
\end{aligned}
$$


Then, for $s>0$

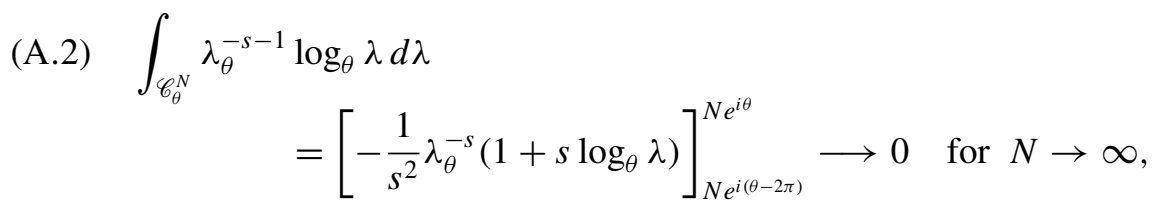
since $N^{-s}$ and $N^{-s} \log N$ go to 0 for $N \rightarrow \infty$. It follows that

$$
\lim _{s \searrow 0} \lim _{N \rightarrow \infty} \int_{\mathscr{C}_{\theta}^{N}} \lambda_{\theta}^{-s-1} \log _{\theta} \lambda d \lambda=0 .
$$

Observe that the order of the limits is important.

Using the resolvent identity $A(A-\lambda)^{-1}=1+\lambda(A-\lambda)^{-1}$ we now get for $x \in D(A)$ :

(A.4)

$$
\begin{aligned}
\lim _{s \searrow 0} \int_{\mathscr{C}_{\theta}} \lambda_{\theta}^{-s} \log _{\theta} \lambda(A-\lambda)^{-1} x d \lambda \\
\quad=\lim _{s \searrow 0} \lim _{N \rightarrow \infty} \int_{\mathscr{C}_{\theta}^{N}} \lambda_{\theta}^{-s} \log _{\theta} \lambda(A-\lambda)^{-1} x d \lambda \\
\quad=\lim _{s \searrow 0} \lim _{N \rightarrow \infty}\left[\int_{\mathscr{C}_{\theta}^{N}} \lambda_{\theta}^{-s-1} \log _{\theta} \lambda x d \lambda+\int_{\mathscr{C}_{\theta}^{N}} \lambda_{\theta}^{-s} \log _{\theta} \lambda(A-\lambda)^{-1} x d \lambda\right] \\
\quad=\lim _{s \searrow 0} \lim _{N \rightarrow \infty} \int_{\mathscr{C}_{\theta}^{N}} \lambda_{\theta}^{-s-1} \log _{\theta} \lambda\left[1+\lambda(A-\lambda)^{-1}\right] x d \lambda \\
\quad=\lim _{s \searrow 0} \lim _{N \rightarrow \infty} \int_{\mathscr{C}_{\theta}^{N}} \lambda_{\theta}^{-s-1} \log _{\theta} \lambda A(A-\lambda)^{-1} x d \lambda,
\end{aligned}
$$

where we used (A.3) in the second line (adding zero). Then, since $\left\|(A-\lambda)^{-1}\right\| \dot{\leq}|\lambda|^{-1}$,

$$
\left\|\lambda_{\theta}^{-s-1} \log _{\theta} \lambda A(A-\lambda)^{-1} x\right\| \dot{\leq}|\log \lambda||\lambda|^{-s-2}\|A x\|,
$$

so that the integrand in the last expression of (A.4) is integrable along $\mathscr{C}_{\theta}$ uniformly in $s>0$, and

$$
\begin{aligned}
& \text { (A.6) } \lim _{s \searrow 0} \lim _{N \rightarrow \infty} \int_{\mathscr{C}_{\theta}^{N}} \lambda_{\theta}^{-s-1} \log _{\theta} \lambda A(A-\lambda)^{-1} x d \lambda \\
& =\int_{\mathscr{C}_{\theta}} \lambda^{-1} \log _{\theta} \lambda A(A-\lambda)^{-1} x d \lambda .
\end{aligned}
$$

Combining (A.4) and (A.6) (and multiplying with $\frac{i}{2 \pi}$ ) we obtain the desired result (4.6). 
The identity (4.7) stems from [3] (we have corrected a sign here). For this, consider the integration contour

(A.7) $\Gamma_{\theta, \varphi}^{N}=\left\{r e^{i \varphi} \mid N>r>r_{0}\right\}$

$$
\cup\left\{r_{0} e^{i \omega} \mid \varphi \geq \omega \geq \theta\right\} \cup\left\{r e^{i \theta} \mid r_{0}<r<N\right\} .
$$

Using again $A(A-\lambda)^{-1}=1+\lambda(A-\lambda)^{-1}$ we obtain

(A.8) $\int_{\Gamma_{\theta, \varphi}^{N}} \lambda^{-1} A(A-\lambda)^{-1} x d \lambda=\int_{\Gamma_{\theta, \varphi}^{N}}(A-\lambda)^{-1} x d \lambda+\int_{\Gamma_{\theta, \varphi}^{N}} \lambda^{-1} x d \lambda$.

For the second term we have, using a logarithm with branch cut disjoint from $\Lambda_{\theta, \varphi}$,

$$
\int_{\Gamma_{\theta, \varphi}^{N}} \lambda^{-1} d \lambda=[\log \lambda]_{N e^{i \varphi}}^{N e^{i \theta}}=i(\theta-\varphi)
$$

Thus

$$
\text { (A.10) } \frac{i}{2 \pi} \int_{\Gamma_{\theta, \varphi}^{N}} \lambda^{-1} A(A-\lambda)^{-1} x d \lambda=\frac{i}{2 \pi} \int_{\Gamma_{\theta, \varphi}^{N}}(A-\lambda)^{-1} x d \lambda+\frac{\varphi-\theta}{2 \pi} x \text {. }
$$

For $x \in D(A)$ the limit for $N \rightarrow \infty$ is well-defined on the left-hand side, and the limit of the first term on the right-hand side then exists as an improper integral, as indicated.

Proof of Lemma 4.3. The integral along $\mathscr{C}_{\theta}$ is, in detail: (A.11)

$$
\begin{aligned}
\int_{\mathscr{C}_{\theta}} \log _{\theta} \lambda f(\lambda) d \lambda=\int_{\infty}^{r_{0}}(\log r+i \theta) f\left(r e^{i \theta}\right) e^{i \theta} d r \\
+\int_{\theta}^{\theta-2 \pi}\left(\log r_{0}+i \omega\right) f\left(r_{0} e^{i \omega}\right) i r_{0} e^{i \omega} d \omega \\
+\int_{r_{0}}^{\infty}(\log r+i \theta-2 \pi i) f\left(r e^{i \theta-2 \pi i}\right) e^{i \theta-2 \pi i} d r .
\end{aligned}
$$

Since $f\left(r e^{i \theta-2 \pi i}\right) e^{i \theta-2 \pi i}=f\left(r e^{i \theta}\right) e^{i \theta}$, the two terms with $(\log r+i \theta)$ cancel each other. Thus

$$
\text { (A.12) } \begin{aligned}
& \int_{\mathscr{C}_{\theta}} \log _{\theta} \lambda f(\lambda) d \lambda \\
= & -\int_{\theta-2 \pi}^{\theta}\left(\log r_{0}+i \omega\right) f\left(r_{0} e^{i \omega}\right) i r_{0} e^{i \omega} d \omega-2 \pi i \int_{r_{0}}^{\infty} f\left(r e^{i \theta}\right) e^{i \theta} d r .
\end{aligned}
$$


Denote the integrand in the first integral $g(\omega)=\left(\log r_{0}+i \omega\right) f\left(r_{0} e^{i \omega}\right) i r_{0} e^{i \omega}$.

There is of course an identity similar to (A.12) with $\theta$ replaced by $\varphi$, and then

$$
\begin{aligned}
\int_{\mathscr{C}_{\theta}} \log _{\theta} \lambda f(\lambda) d \lambda-\int_{\mathscr{C}_{\varphi}} \log _{\varphi} \lambda f(\lambda) d \lambda \\
=\left(-\int_{\theta-2 \pi}^{\theta}+\int_{\varphi-2 \pi}^{\varphi}\right) g(\omega) d \omega \\
\quad-2 \pi i\left(\int_{r_{0}}^{\infty} f\left(r e^{i \theta}\right) e^{i \theta} d r-\int_{r_{0}}^{\infty} f\left(r e^{i \varphi}\right) e^{i \varphi} d r\right) \\
=\left(-\int_{\theta-2 \pi}^{\theta}+\int_{\varphi-2 \pi}^{\varphi}\right) g(\omega) d \omega \\
\quad-2 \pi i \int_{\infty}^{r_{0}} f\left(r e^{i \varphi}\right) e^{i \varphi} d r-2 \pi i \int_{r_{0}}^{\infty} f\left(r e^{i \theta}\right) e^{i \theta} d r .
\end{aligned}
$$

The last two terms are recognized as the contributions to $-2 \pi i \int_{\Gamma_{\theta, \varphi}} f(\lambda) d \lambda$ from the rays $e^{i \varphi}\left[r_{0}, \infty\left[\right.\right.$ and $e^{i \theta}\left[r_{0}, \infty[\right.$. The first term is seen to give the contribution from the $\operatorname{arc} \mathscr{C}_{r_{0}, \theta, \varphi}=\left\{r_{0} e^{i \omega} \mid \varphi \geq \omega \geq \theta\right\}$ as follows:

$$
\begin{aligned}
\left(-\int_{\theta-2 \pi}^{\theta}+\int_{\varphi-2 \pi}^{\varphi}\right) g(\omega) d \omega & \\
= & \left(-\int_{\varphi}^{\theta}+\int_{\varphi-2 \pi}^{\theta-2 \pi}\right) g(\omega) d \omega=\int_{\varphi}^{\theta}[-g(\omega)+g(\omega-2 \pi)] d \omega \\
= & \int_{\varphi}^{\theta}\left[-\left(\log r_{0}+i \omega\right) f\left(r_{0} e^{i \omega}\right) i r_{0} e^{i \omega}\right. \\
& \left.\quad+\left(\log r_{0}+i(\omega-2 \pi)\right) f\left(r_{0} e^{i \omega}\right) i r_{0} e^{i \omega}\right] d \omega \\
= & -2 \pi i \int_{\varphi}^{\theta} f\left(r_{0} e^{i \omega}\right) i r_{0} e^{i \omega} d \omega=-2 \pi i \int_{\mathscr{C}_{r_{0}, \theta, \varphi}} f(\lambda) d \lambda .
\end{aligned}
$$

\section{REFERENCES}

1. Atiyah, M. F., Patodi, V. K., and Singer, I. M., Spectral asymmetry and Riemannian geometry, I, Math. Proc. Cambridge Philos. Soc. 79 (1976), 43-69.

2. Boutet de Monvel, L., Boundary problems for pseudo-differential operators, Acta Math. 126 (1971), 11-51.

3. Burak, T., On spectral projections of elliptic operators, Ann. Scuola Norm. Sup. Pisa (3) 24 (1970), 209-230. 
4. Fedosov, B. V., Golse, F., Leichtnam, E., Schrohe, E., The noncommutative residue for manifolds with boundary, J. Funct. Anal. 142 (1996), 1-31.

5. Gilkey, P. B., The residue of the global eta function at the origin, Adv. Math. 40 (1981), 290-307.

6. Grubb, G., Functional calculus of pseudodifferential boundary problems, Progr. Math. 65, Second Edition, 1996, first edition issued 1986.

7. Grubb, G. Spectral boundary conditions for generalizations of Laplace and Dirac operators, Comm. Math. Phys. 240 (2003), 243-280.

8. Grubb, G., On the logarithm component in trace defect formulas, Comm. Partial Differential Equations 30 (2005), 1671-1716.

9. Grubb, G., The local and global parts of the basic zeta coefficient for pseudodifferential boundary operators, Math. Ann. 341 (2008), 735-788.

10. Grubb, G., and Hörmander, L., The transmission property, Math. Scand. 67 (1990), 273-289.

11. Guillemin, V., A new proof of Weyl's formula on the asymptotic distribution of eigenvalues, Adv. Math. 102 (1985), 184-201.

12. Kato, T., Perturbation Theory for Linear Operators, Springer Verlag, Berlin, 1966.

13. Okikiolu, K., The Campbell-Hausdorff theorem for elliptic operators and a related trace formula, Duke Math. J. 79 (1995), 687-722.

14. Ponge, R., Spectral asymmetry, zeta functions, and the noncommutative residue, Internat. J. Math. 17 (2006), 1065-1090.

15. Scott, S., The residue determinant, Comm. Partial Differential Equations 30 (2005), 483-507.

16. Seeley, R. T., Complex powers of an elliptic operator, in "Singular Integrals", Proc. Sympos. Pure Math. X, 1967, 288-307.

17. Seeley, R. T., The resolvent of an elliptic boundary problem, Amer. J. Math. 91 (1969), 889-920.

18. Seeley, R. T., Norms and domains of the complex powers $A_{B}^{s}$, Amer. J. Math. 93 (1971), 299-309.

19. Wodzicki, M., Spectral asymmetry and zeta functions, Invent. Math. 66 (1982), 115-135.

20. Wodzicki, M., Local invariants of spectral asymmetry, Invent. Math. 75 (1984), 143-178.

COPENHAGEN UNIVERSITY

MATHEMATICS DEPARTMENT

UNIVERSITETSPARKEN 5

DK-2100 COPENHAGEN

DENMARK

E-mail: gaarde@math.ku.dk, grubb@math.ku.dk 